\title{
MOCCA-SURVEY database I. Accreting white dwarf binary systems in globular clusters - II. Cataclysmic variables - progenitors and population at birth
}

\author{
Diogo Belloni ${ }^{1,2 \star}$ Mirek Giersz ${ }^{1} \dagger$, Helio J. Rocha-Pinto ${ }^{3}$, Nathan W. C. Leigh $^{4}$, \\ Abbas Askar ${ }^{1}$ \\ ${ }^{1}$ Nicolaus Copernicus Astronomical Centre, Polish Academy of Sciences, ul. Bartycka 18, PL-00-716 Warsaw, Poland \\ 2 CAPES Foundation, Ministry of Education of Brazil, DF 70040-020, Brasilia, Brazil \\ ${ }^{3}$ Universidade Federal do Rio de Janeiro, Observatório do Valongo, Ladeira do Pedro Antônio 43, 20080-090 Rio de Janeiro, Brazil \\ ${ }^{4}$ Department of Astrophysics, American Museum of Natural History, Central Park West and 79th Street, New York, NY 10024, USA
}

Accepted 2016 September 30. Received 2016 September 28; in original form 2016 August 26

\begin{abstract}
This is the second in a series of papers associated with cataclysmic variables (CVs) and related objects, formed in a suite of simulations for globular cluster evolution performed with the MOCCA Monte Carlo code. We study the properties of our simulated CV populations throughout the entire cluster evolution. We find that dynamics extends the range of binary $\mathrm{CV}$ progenitor properties, causing $\mathrm{CV}$ formation from binary progenitors that would otherwise not become CVs. The CV formation rate in our simulations can be separated into two regimes: an initial burst $(\lesssim 1 \mathrm{Gyr}$ ) connected with the formation of the most massive white dwarfs, followed by a nearly constant formation rate. This result holds for all models regardless of the adopted initial conditions, even when most CVs form dynamically. Given the cluster agedependence of $\mathrm{CV}$ properties, we argue that direct comparisons to observed Galactic field CVs could be misleading, since cluster CVs can be up to 4 times older than their field counterparts. Our results also illustrate that, due mainly to unstable mass transfer, some CVs that form in our simulations are destroyed before the present-day. Finally, some field CVs might have originated from globular clusters, as found in our simulations, although the fraction of such escapers should be small relative to the entire Galactic field CV population.
\end{abstract}

Key words: novae, cataclysmic variables - globular clusters: general - methods: numerical

\section{INTRODUCTION}

Cataclysmic variables (CVs) are interacting binaries composed of a white dwarf (WD) undergoing stable mass transfer from a main sequence (MS) star or a brown dwarf (BD) (e.g. Warner 1995; Knigge et al. 2011). They are expected to exist in non-negligible numbers in globular clusters (GCs), that are natural laboratories for testing theories of stellar dynamics and evolution.

CVs in GCs have been studied by many authors, both theoretically and observationally (e.g. Knigge 2012, and references therein). GCs are thought to play a crucial role in $\mathrm{CV}$ formation, since their densities are sufficiently high that dynamical encounters involving binaries should be common. Thus, in dense GCs, it is natural to expect that many $\mathrm{CV}$ progenitors will have been affected by dynamics in some way prior to $\mathrm{CV}$ formation (e.g. Ivanova et al. 2006; Belloni et al. 2016).

\footnotetext{
* E-mail: belloni@camk.edu.pl (DB)

$\dagger$ E-mail: mig@camk.edu.pl (MG)
}

\subsection{Formation and Destruction Channels}

The primary channels associated with CV formation in GCs, based on the results of numerical simulations, can be summarized as (Ivanova et al. 2006) following: (i) only $\sim 27$ per cent of CVs form from binaries that have never experienced a dynamical interaction; (ii) $\sim 60$ per cent of CVs did not evolve via a commonenvelope phase (CEP); (iii) tidal capture does not play a significant role in CV formation; and (iv) dynamical encounters tend to exchange more massive WDs into the binary progenitors of CVs.

Some of these formation channels have been independently discussed by Shara \& Hurley (2006). The authors analyzed two cluster $N$-body models, simulated using the NBODY4 code with GRAPE-6 processors. In their simulations, they found 4 (out of 15) $\mathrm{CV}$ s had no field-like counterpart, i.e. four CVs were dynamically formed, either by exchange or successive dynamical encounters.

In both Ivanova et al. (2006) and Shara \& Hurley (2006), the authors also discussed destruction channels for any CVs that do not survive to the present-day in their simulations. Ivanova et al. (2006) found that most CVs cease mass transfer for (internal) evolution- 


\section{Belloni et al.}

ary reasons; very few CVs are destroyed by dynamical encounters. Shara \& Hurley (2006) found similar results in this regard, however fewer CVs managed to escape from their simulated clusters relative to what was found by Ivanova et al. (2006), and the mechanism of escape was always weak encounters or two-body relaxation.

In spite of the agreement between these two modeling efforts, we emphasize that Shara \& Hurley (2006) analyzed only two models (100k and 200k) and had very few CVs form $(<20)$, whereas Ivanova et al. (2006) analyzed several Monte Carlo models with many CVs, but lacked any cluster evolution, i.e. their clusters had fixed spatial structure. We aim to complement these pioneering works with the MOCCA Monte Carlo simulations presented here, which offer both statistical significance and a realistic dynamical environment for the host clusters.

\subsection{Dynamical Influence on CV formation and evolution}

One interesting feature described by Shara \& Hurley (2006) is a dynamically-induced acceleration in the onset and rate of accretion when CVs are formed. For example, the authors discuss a particular $\mathrm{CV}$ formed in their simulations that began the $\mathrm{CV}$ phase before its field-like counterpart, due to a prior dynamical interaction. Another CV had its evolution accelerated by a dynamical perturbation just after initiating the $\mathrm{CV}$ phase.

We argue that the first of these two scenarios is more likely, since $\mathrm{CV}$ progenitors tend to be more massive than the mean stellar and even binary mass in the cluster, which reduces the time-scale for dynamical encounters prior to the onset of the $\mathrm{CV}$ phase. If these interactions decrease the binary progenitor orbital period (or increase its eccentricity), then the CE phase will begin earlier than for its field counterpart. This scenario would not necessarily rely on a single strong interaction, but could rather arise due to repeated weak interactions.

The second scenario, on the other hand, seems much less likely, since this requires (very few) strong dynamical interactions and hence small impact parameters. CVs tend to have short orbital periods, which reduces the probability for direct encounters with sufficient force to significantly change their orbital parameters (Leigh et al. 2016). However, if the CV is formed close to or in the cluster core, then the probability of a strong interaction is at its highest. This was the case for the specific CV that suffered accelerated mass transfer due to a dynamical perturbation in the simulations of Shara \& Hurley (2006), since this binary was very close to the core at its formation.

We further caution the reader that these conclusions taken from Shara \& Hurley (2006) rely on small number CV statistics, albeit accompanied by rich details for their formation and subsequent time evolution. Hence, we emphasize that these results need to be confirmed, by supplanting the small number statistics with a more robust coverage of the relevant parameter space for GC evolution and CV formation, using a much larger suite of realistic simulations.

\subsection{CV age}

We emphasize that observing CVs in GCs relies on much more than just dynamical interactions. Of comparable or even greater importance are the observational selection effects, as well as the ages of cluster CVs compared to Galactic field CVs. For instance, Belloni et al. (2016) quantified the observational selection effects that plague the search for CVs in GCs, and concluded that their detection rates could be dramatically increased if detectable during quiescence. Ak et al. (2015) inferred the ages of a sample of field $\mathrm{CVs}$ from kinematic data, and concluded that 94 per cent of CVs in the solar neighbourhood belong to the thin-disc component of the Galaxy. The corresponding mean kinematical ages are $3.40 \pm$ $1.03 \mathrm{Gyr}$ and $3.90 \pm 1.28 \mathrm{Gyr}$ for the non-magnetic thin-disc CVs below and above the period gap, respectively. In GCs, on the other hand, some CVs can be up to 4 times older than this. Thus, it is critical to properly account for such age-related effects when comparing cluster and field CV populations, in an attempt to quantify the impact of the cluster dynamics on $\mathrm{CV}$ formation and evolution.

\subsection{Structure of the paper}

For clarity, we have separated the results of our initial investigation into CV formation in GCs into two different papers. In the first paper in this series (Belloni et al. 2016), we concentrated on the present-day population (PDP) of CVs and the observational selection effects that contribute to "hiding" most of the CV populations in GCs from observations.

In this paper, the second of the series, we focus on the primary formation channels for CVs, as simulated by the MOCCA code (Section 2.1), and quantify the influence of the cluster dynamics in shaping the observed CV properties. We further address the agedependence of $\mathrm{CV}$ properties, CVs destroyed before the presentday (i.e., after $12 \mathrm{Gyr}$ of cluster evolution) and CVs formed from binary progenitors that previously escaped their host cluster.

In Section 2, we describe the MOCCA and CATUABA codes and present the suite of models analyzed in this paper. In Section 3, the main results of this investigation are presented and discussed. We conclude and summarize our main results in Section 4.

\section{METHODOLOGY AND MODELS}

In this section, we briefly describe the MOCCA code that was used to simulate the six cluster realizations considered here, the CATUABA code that was used to analyze the simulated CV populations in each model and, finally, the initial cluster conditions.

\subsection{MOCCA code}

The MOCCA code is based on the orbit-averaged Monte Carlo technique for cluster evolution developed by Hénon (1971), which was further improved by Stodółkiewicz (1986), and then developed even further by Giersz et al. (2013, and references therein). These last authors included in MOCCA the FEWBODY code, developed by Fregeau et al. (2004) to perform numerical scattering experiments of small-number gravitational interactions. To model the Galactic potential, MOCCA assumes a point-mass with total mass equal to the enclosed Galaxy mass at the specified Galactocentric radius. The description of escape processes in tidally limited clusters follows the procedure derived by Fukushige \& Heggie (2000). The stellar evolution is implemented via the SSE code developed by Hurley et al. (2000) for single stars and the BSE code developed by Hurley et al. (2002) for binary evolution.

The most important part of MOCCA with regards to CV formation and related exotic objects is the BSE code (Hurley et al. 2002). Here, as in Belloni et al. (2016), we adopt the standard parameters described in Hurley et al. (2002), which includes the adoption of their assumed efficiency parameter for the CE phase 
$(\alpha=3)^{1}$. We emphasize that the defaults in BSE, and especially the assumption for $\alpha$, may be over-estimates of realistic values (e.g. Zorotovic et al. 2010; Camacho et al. 2014). However, our focus in this paper is to quantify the role of cluster dynamics and evolution on $\mathrm{CV}$ properties in globular clusters. We defer a more thorough investigation of the various aspects of binary evolution relevant for $\mathrm{CV}$ formation to a forthcoming paper. For now, we note simply that our adopted assumptions for the BSE code should mainly affect the total numbers of CVs formed via binary evolution (in particular the CEP). Our conclusions regarding the influence of the cluster dynamics on $\mathrm{CV}$ formation should be roughly insensitive to these assumptions.

MOCCA has been extensively tested against N-body codes. For instance, Giersz et al. (2013) showed that MOCCA reproduces the results of $N$-body codes with excellent precision, including not only the rate of cluster evolution and the subsequent re-structuring of the cluster mass distribution, but also the calculated distributions of binary orbital parameters. Additionally, Wang et al. (2016) compared MOCCA with the state-of-the-art NBODY6++GPU, showing good agreement between the predictions of the two codes.

To summarize, due to its fast, efficient and accurate coverage of the relevant parameter space, MOCCA is ideal for performing big surveys aimed at modeling large populations of CVs in many GCs (Leigh et al. 2013, 2015; Giersz et al. 2015), and for studying in detail the influence of the host cluster environment on the properties of different types of exotic objects such as CVs (Belloni et al. 2016, this work), blue straggler stars (Hypki \& Giersz 2013, 2016a,b), intermediate-mass black holes (Giersz et al. 2015), X-ray binaries, etc.

\subsection{CATUABA code}

The CATUABA (Code for Analyzing and sTUdying cAtaclysmic variables, symBiotic stars and AM CVns) code was introduced and described in Belloni et al. (2016). The code was created for the purpose of analyzing populations of CVs and related objects (AM CVns and symbiotic stars) produced in clusters simulated by the MOCCA code.

In its current version, it allows for a detailed analysis of the entire CV population produced over the course of the simulated cluster evolution. It combines the BSE code with the disc instability model (DIM) constrained by empirical data (in order to fill in any gaps in binary evolution not accounted for in BSE) to calculate the observed CV properties.

CATUABA permits for the unambiguous identification of a given $\mathrm{CV}$ population in the cluster snapshots provided by MOCCA at various times in the cluster evolution. It then compiles the most relevant events (related to both stellar and binary evolution, as well as dynamical interactions) in the history of all individual CVs. Armed with this information, CATUABA unequivocally separates the CVs into three groups, defined by the influence of dynamical interactions incurred over the course of the binary progenitor lifetimes. The first group contains only CVs formed without any influence from dynamics (BSE group), the second group includes CVs weakly influenced by dynamics (WDI group), and the last group

1 BSE assumes that the common envelope binding energy is that of the giant(s) envelope involved in the process. In order to reconcile the prescription developed by Iben \& Livio (1993), the recommended value for the CEP efficiency is 3.0. includes those CVs strongly influenced by dynamical interactions (SDI group).

Some comments are worth mentioning here regarding the distinction between the WDI and SDI groups (Belloni et al. 2016, see section 3.2 for more details):

(i) Whether a CV belongs to the WDI group or the SDI group is a priori arbitrary. We define this transition based on the fraction of energy exchanged between the target binary and interloping single or binary star at the end of a dynamical interaction, relative to the initial orbital energy of the target binary. The exact cutoff between weak and strong dynamical interactions is 20 per cent of the initial binding energy. We emphasize that this cutoff applies only on a per interaction basis, and is an arbitrarily chosen value based on the average energy exchange given in Spitzer's formula for equal-mass systems (Spitzer 1987). Generally, for the WDI group, most of the interactions are fly-bys. However, resonant interactions do sometimes occur, although relatively rarely by comparison. For the SDI group, most interactions are resonant and/or exchanges. According to our definition, a binary experiences an WDI if it undergoes a single interaction and incurs a change in its binding energy that is less than 20 per cent of its initial value. Otherwise, the binary was subjected to an SDI. If a given binary experiences only weak dynamical interactions over the course of its lifetime, then this binary belongs to the WDI group. Conversely, if the binary underwent one or more strong dynamical interactions over the course of its lifetime, then this binary belongs to the SDI group.

(ii) The WDI group is formed by CVs that had only weak encounters during their lives. However, if the number of these encounters is high for a $\mathrm{CV}$ in this group, their cumulative effect can be strong and the properties of the progenitor binary can be strongly changed during its life. Otherwise, if the cumulative effect of the weak dynamical interactions is not strong, the CV will probably have similar properties to those belonging to the BSE group.

(iii) Only the SDI group includes binaries that underwent either exchange or merger.

The CATUABA code also provides information regarding the progenitor binary populations, and the formation-age populations. The progenitor population is defined as the population of all binaries that are $\mathrm{CV}$ progenitors, i.e. the population of ' $\mathrm{CVs}$ ' at the time of cluster birth (i.e., $t=0$ ). The formation-age population is defined as the population of binary progenitors at the time of initiation of the CV phase (i.e., when mass transfer starts from the donor on to the WD). Obviously, for the formation-age population, this time is not unique, since the time corresponding to the onset of the $\mathrm{CV}$ phase is different for every $\mathrm{CV}$.

Finally, CATUABA allows for the study of CVs that form in the cluster but do not survive up to the present-day (i.e., destroyed $\mathrm{CVs}$ ), while also for the clear identification of $\mathrm{CVs}$ formed from escaped binaries (for ease of comparison to analogous field CV populations formed from primordial binaries born in the field). In short, CATUABA identifies, organizes and quantifies the time evolution of CV properties in realistic evolving GCs.

All features and main assumptions concerning the CATUABA code are explained in more detail in Belloni et al. (2016, see their section 3$)$.

\subsection{Models}

In this work, we continue the analysis started by Belloni et al. (2016), who investigated models with two distinct initial binary populations (IBPs). Each IBP contains all initial binaries in a given 
cluster, and is associated with a specific set of binary orbital parameter distributions. Briefly, the six models analyzed in Belloni et al. (2016) differ mainly with respect to the initial central density, the initial binary fraction and choice of the IBP.

The first set of models, defined as the K (or Kroupa) models, correspond to models with 95 per cent primordial binaries, with the IBPs having binary orbital parameter distributions that follow the Kroupa IBP (Kroupa 2008), which is derived from eigenevolution and mass feeding algorithms (Kroupa 2008).

The second set of models, defined as the S (or Standard) models, correspond to models with 5 or 10 per cent primordial binaries, with the IBPs having binary orbital parameter distributions that follow the Standard IBP. The Standard IBP follows a uniform distribution for the binary mass ratio, an uniform distribution in the logarithm of the semi-major axis $\log (a)$ or a log-normal semimajor axis distribution, and a thermal eccentricity distribution. For these two sets of models, we consider sparse $\left(\rho_{c} \sim 10^{3} \mathrm{M}_{\odot} \mathrm{pc}^{-3}\right)$, dense $\left(\rho_{c} \sim 10^{5} \mathrm{M}_{\odot} \mathrm{pc}^{-3}\right)$, and very dense $\left(\rho_{c}>10^{5} \mathrm{M}_{\odot} \mathrm{pc}^{-3}\right)$ clusters, where $\rho_{c}$ is the cluster central density.

We assume that all stars are on the zero-age main sequence when the simulation begins, and that all residual gas remaining from the star formation process has already been removed from the cluster at $\mathrm{t}=0$. All models adopt a low metallicity $(\lesssim 0.001)$, are initially in virial equilibrium, and have neither rotation nor primordial mass segregation. Finally, all models are evolved up to a cluster age of $12 \mathrm{Gyr}$, which is taken to be the "present-day".

The properties of all initial models are summarized in Table 1 of Belloni et al. (2016). We have also evolved these same models (actually, just the IBP) with BSE alone over a $12 \mathrm{Gyr}$ period. In this way, we create a "control" sample of CVs, for comparison to the cluster CVs formed in our Monte Carlo simulations. This control sample is therefore tailored for comparison to observed CV populations in the Galactic field. This will help us to disentangle the role of the cluster dynamics in shaping the properties of $\mathrm{CV}$ populations in GCs, for all six initial models considered here.

After describing the main codes performing the required calculations and the six sets of initial conditions considered, in the next section we turn our attention to the results of our analysis of these models. We begin by presenting our main results concerning the progenitor (i.e., initial) and formation-age (i.e., at the onset of the $\mathrm{CV}$ phase) populations that ultimately produced the CVs observed in our simulations at the present-day. We further describe the agedependences found for the properties of our simulated CVs, including effects related to $\mathrm{CV}$ destruction and escape (from the cluster).

\section{RESULTS AND DISCUSSION}

In the first paper (Belloni et al. 2016), we were interested predominantly in the properties of the PDPs of CVs and the related observational selection effects. In this paper, we concentrate on the influence of the cluster dynamics in shaping the observed CV PDPs. We further consider the evolution of our CV populations throughout the entire cluster lifetime to quantify the predicted agedependent properties of CVs in GCs.

We begin with a brief summary of the expected present-day CV properties in GCs. Belloni et al. (2016, see their table 2) provide detailed information regarding the influence of dynamics in $\mathrm{CV}$ formation, for CVs that form during the cluster evolution and survive to the present-day. Their results can be summarized as follows:

(i) all CVs formed in models that adopt a Kroupa IBP have a dy- namical origin; exchange interactions are the most important channel for CV formation, which are responsible for $\sim 2 / 3$ of the entire PDP of CVs;

(ii) for sparse clusters, in particular the $\mathrm{S} 1$ and $\mathrm{K} 1$ models, 'dynamical' formation channels occur very rarely;

(iii) dynamically formed CVs tend to have more massive WDs, due to exchange interactions that preferentially eject low-mass objects;

(iv) $\mathrm{CV}$ s formed without any influence from the cluster dynamics tend to have less massive WDs $\left(\sim 0.3 \mathrm{M}_{\odot}\right)$;

(v) $\sim 87$ per cent of the CVs formed in our simulations are period bouncers, with extremely low-mass donors $\left(\lesssim 0.05 \mathrm{M}_{\odot}\right)$.

In the subsequent sections, we elaborate and expand these results. We first state the main properties of, and discuss the influence of dynamics on, the progenitor populations (Section 3.1). Then, we outline the main channels for $\mathrm{CV}$ formation and compare our results to those of Ivanova et al. (2006) (Section 3.2). To address the importance of the global cluster evolution for CV formation, in Section 3.3 we show that the rate of CV formation is roughly independent of the cluster configuration throughout its entire lifetime. We present the formation-age population properties in Section 3.4, and discuss the main implications of these properties for observations of CVs in GCs. This leads to a discussion of the influence of dynamics on $\mathrm{CV}$ evolution, from $\mathrm{CV}$ birth until the present-day (Section 3.5). We go on to discuss the time-dependent properties of $\mathrm{CV}$ populations in GCs, pointing out the expected differences between observations of Galactic field and cluster CVs (Section 3.7). Finally, we discuss those CVs formed throughout the cluster evolution, but destroyed or ejected from the cluster before the present-day (Section 3.8) and CVs formed from binary progenitors that previously escaped their host cluster (Section 3.9).

\subsection{Progenitor Population}

The progenitor population is identified in CATUABA as follows. For CV progenitors that never undergo exchanges the components of the primordial progenitor binary are always the same as the components of the $\mathrm{CV}$, so that they are easily identified. However, the identification is not as straightforward when an exchange encounter has occurred. Here, the initial binary properties are obtained by first identifying the properties of the binary with the smaller period (if an exchange took place via a binary-binary interaction) or simply the properties of the initial binary (if an exchange happened via a single-binary interaction). In the event that both binary components of a given $\mathrm{CV}$ in the PDP were originally single stars at the time of cluster birth, then the $\mathrm{CV}$ is excluded from inclusion in the progenitor population, since there is no associated initial binary (Belloni et al. 2016, see Section 3.1.2 for more details).

Fig. 1 shows the distributions of the WD progenitor masses, mass ratios $(\leqslant 1)$ and periods of the progenitor $\mathrm{CV}$ population, divided according to the subgroups defined in Belloni et al. (2016, see section 3.2).

In general, $\mathrm{CV}$ progenitors from the BSE group occupy a smaller volume in the parameter space defined by the orbital period, eccentricity, primary mass and mass ratio relative to the WDI and SDI groups. This is because dynamical interactions are able to trigger $\mathrm{CV}$ formation in binaries that otherwise would never undergo a CV phase, as illustrated in the bottom row of each panel in Fig. 1.

We turn now to a more detailed description of the binary prop- 
a

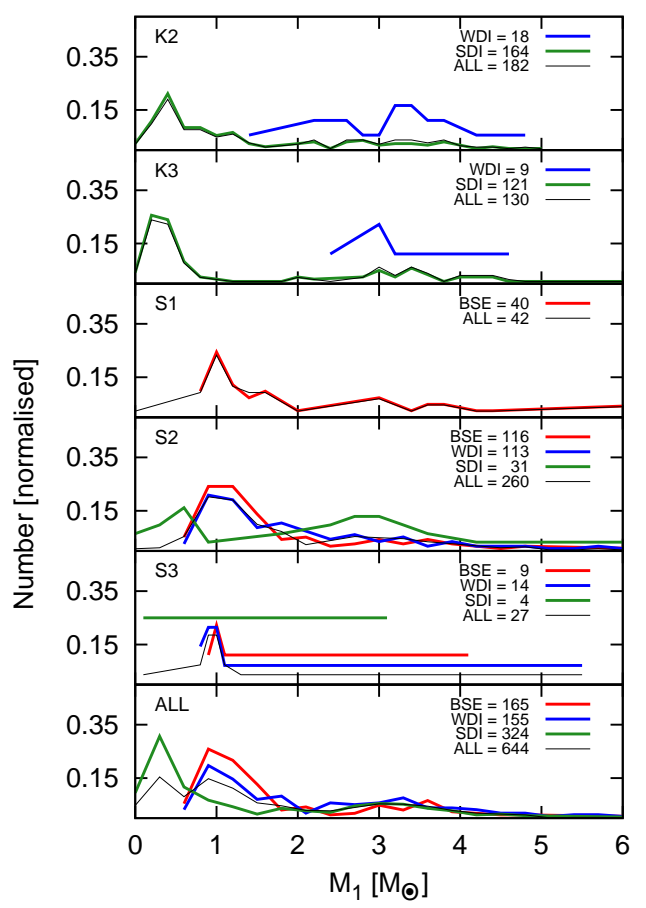

b

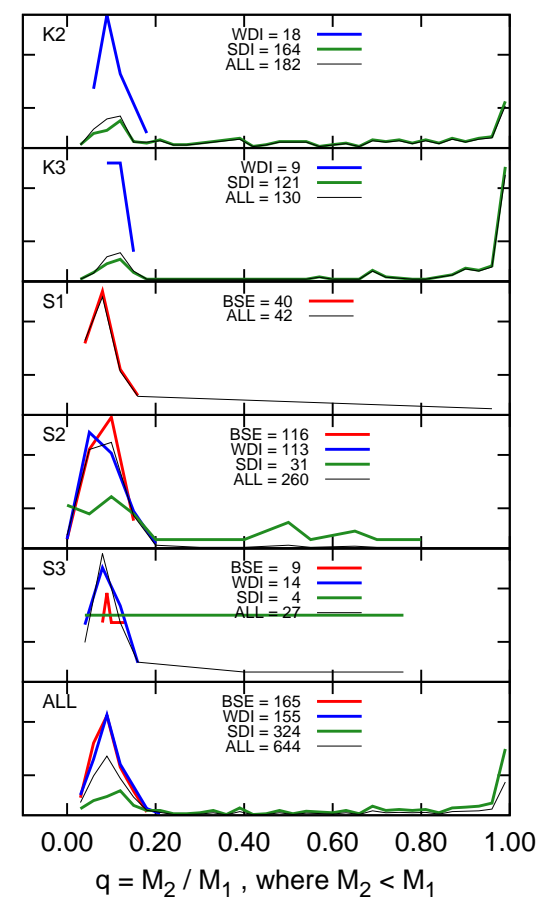

C

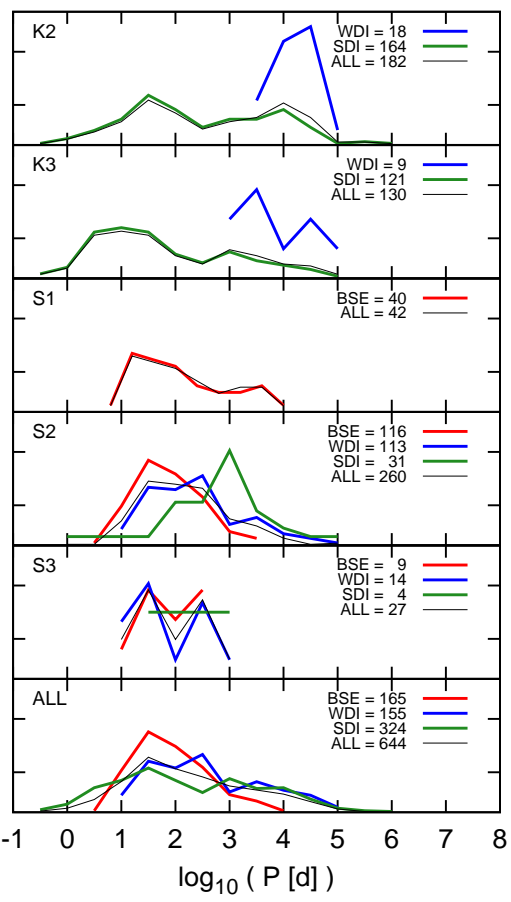

Figure 1. Primary mass (left-hand panel), mass ratio (middle panel), and period (right-hand panel) distributions for progenitor binaries that become CVs and survive to the present-day, shown for all models (model K1 is not shown because only three CVs form and survive up to 12 Gyr in this model). The keys indicate the number of CVs in each model and group. The bottom row presents the progenitor CV populations in all six models aggregated into a single sample. From the bottom row of each graph, we see a clear extension in the range of binary parameters relevant to CV formation, due to pure binary stellar evolution only (BSE group) being influenced by dynamical interactions (WDI and SDI groups). For more details, see Section 3.1.

erties characteristic of the progenitor populations. For simplicity, we consider binaries from all six models aggregated into a single distribution (bottom row of each panel in Fig. 1), unless otherwise stated.

\subsubsection{Primary Mass}

Binaries in the BSE and WDI groups are quite similar in terms of their primary mass (i.e., WD progenitor) distributions (Fig. 1a). This indicates that weak dynamical interactions are not important in changing the mass of the primary. More precisely, the primary masses (at $t=0$ ) in the BSE and WDI groups are always more massive than $0.8 \mathrm{M}_{\odot}$, with a peak at around $1.0 \mathrm{M}_{\odot}$ and a long tail towards higher masses (up to $\sim 7.0 \mathrm{M}_{\odot}$ ). This lower limit for the primary mass (i.e., WD progenitor at $\mathrm{t}=0$ ) indirectly reflects the turn-off mass evolution, from $t=0$ to $t=12 \mathrm{Gyr}$. As the cluster evolves and MS stars become compact objects due to stellar evolution, the cluster turn-off mass shifts toward lower masses. At 12 Gyr (present-day), the turn-off mass is $\sim 0.8 \mathrm{M}_{\odot}$. This means that MS stars whose masses are lower than $\sim 0.8 \mathrm{M}_{\odot}($ at $t=0)$ will not evolve into WDs within $12 \mathrm{Gyr}$ of stellar evolution.

For binaries in the SDI group, we see primaries with masses $\lesssim 0.8 \mathrm{M}_{\odot}$, with a peak at $\sim 0.5 \mathrm{M}_{\odot}$. As we have just pointed out above, such low-mass MS stars will not evolve into WDs within the Hubble time. Thus, those binaries with primary masses below $0.8 \mathrm{M}_{\odot}($ at $t=0)$ will have at least one component replaced with a more massive star (either a MS star or a WD) (see section 3.2.3) at a time $t>0$. Strictly speaking, for binaries involved in dynamical interactions that lead to exchanges, Fig. 1a does not show the 'real'
WD progenitor at $t=0$. Instead, Fig. 1a shows that for this range of primary masses (at $t=0$ ), the binaries must be involved in at least one exchange interaction (at $t>0$ ) in order to form a 'real' CV progenitor binary. Said another way, Fig. 1a shows that most binaries in the SDI group cannot form CVs as they are at $t=0$. Thus, exchange interactions are needed to convert such binaries (at $t=0$ ) into 'real' CV progenitors (at $t>0$ ). This is especially true for models $\mathrm{K} 2$ and $\mathrm{K} 3$, in which exchange interactions are the principal $\mathrm{CV}$ formation channel. We discuss the role of exchanges in $\mathrm{CV}$ formation in more detail in Section 3.2.3.

\subsubsection{Secondary Mass}

Analogous behaviour is observed between the BSE and WDI groups for the secondary mass distribution (not shown in Fig. 1). The reasons for this are similar as for the primary mass: only strong dynamical interactions can change the component masses.

The masses are, usually, $0.08<M_{2}<1.0 \mathrm{M}_{\odot}$ in the WDI group, with only two cases in which the secondary mass is greater than $1.0 \mathrm{M}_{\odot}$. One of these binaries forms an unstable $\mathrm{CV}$, that later becomes stable due to mass loss.

\subsubsection{Mass ratio}

The mass ratio distribution (Fig. 1b) shows a clear separation between those $\mathrm{CVs}$ formed with a strong dynamical influence and the rest. For binaries in the BSE and WDI groups, the initial mass ratio is always $\lesssim 0.2$. Otherwise, the outcome of the CEP is an unstable $\mathrm{CV}$ with a high probability of merging after a few Myr 
due to an enhanced mass transfer rate. However, in some extremely rare cases, a few $\mathrm{CVs}$ were born unstable and later became stable due to mass loss. In these rare cases, the mass ratio after the CEP is $\sim 1$. Of all six models considered, only one such case emerged, in model S2. Typically, unstable systems do not survive the mass transfer process, as we will see in more detail in Section 3.8.1.

Now, for the CVs in the SDI group, the mass ratio distribution covers the full range, with a slight preference for $\mathrm{q}<0.15$ (in models S2, K2, and K3), and $\mathrm{q}>0.95$ (in models $\mathrm{K} 2$ and $\mathrm{K} 3$ ). This extensive range is due to the fact that binaries can undergo exchanges, which can significantly change their properties. This is clear from Fig. 1b. Additionally, models K2 and K3 show a peak around $\mathrm{q} \sim 1$. This is because the Kroupa models have an initial mass ratio distribution with its peak $\sim 1$.

\subsubsection{Period}

The upper limit of the period distribution (Fig. 1c) for the BSE group is always less than $\sim 10^{4}$ days. This is because secondaries in post-common-envelope binaries (PCEBs) descended from longer period binaries will not fill their Roche-lobes within the Hubble time.

The WDI group contains binaries that suffered only weak dynamical interactions. For these binaries, the initial period can be longer than $10^{4}$ days, if the eccentricity is increased via multiple dynamical interactions. Specifically, the upper period limit can reach $\sim 10^{5}$ days, particularly for models K1 and K2. Such models mostly contain long-period binaries in the WDI group, which indicates that the cumulative effect of weak dynamical interactions is to significantly change the eccentricity, pushing wide binaries to become CVs. On the other hand, models S2 and S3 mostly have short-period binaries, which suggests that the cumulative effect of weak dynamical interactions is insignificant. However, in a few rare cases, this cumulative effect will compound to become significant might be strong (note that a few binaries have periods longer than $\sim 10^{4}$ days in model $\mathrm{S} 2$ ).

The SDI group shows no evidence for a restricted interval in the period distribution, since the binary periods are changed drastically due to strong interactions. We do, however, see an extension of the period interval (compared to the BSE and WDI groups) in the direction of both shorter and longer periods.

\subsubsection{The Progenitor Population}

To sum up, we see a clear correlation between an expansion in the range in $\mathrm{CV}$ progenitor parameter space and the strength of dynamical interactions. In other words, dynamical interactions can bring binaries to the required range in parameter space for $\mathrm{CV}$ formation to occur.

Ivanova et al. (2006) found similar results, although in their Fig. 1, they considered only binaries with circular orbits. This originated from a very restricted IBP, that was evolved without the influence of dynamics. Consequently, we are only able to compare their findings to our results for the BSE group.

\subsection{From progenitor up to formation}

After describing the properties of the progenitor binary population, we now move on to investigating the main formation scenarios leading to $\mathrm{CV}$ formation.
Table 1. Number of CVs present in the clusters at 12 Gyr separated according to their main formation channel. The channels in Ivanova et al. (2006, see their Fig. 7) correspond to the second row. The numbers given in the fourth row indicate the CV formation channel identified in this work. See Section 3.2.1 for more details.

\begin{tabular}{lccccccccc}
\hline \hline \multicolumn{7}{c}{ Formation Channel as defined by Ivanova et al. (2006) } \\
\hline \hline & $1 \mathrm{a}, \mathrm{c}$ & $1 \mathrm{~b}$ & $2 \mathrm{a}$ & $2 \mathrm{~b}$ & $2 \mathrm{c}$ & $3 \mathrm{a}$ & $3 \mathrm{~b}$ & $3 \mathrm{c}$ & \\
\hline \hline Model & 1 & 2 & 3 & 4 & 5 & 6 & 7 & 8 & Total \\
\hline \hline K1 & 0 & 1 & 0 & 0 & 2 & 0 & 0 & 0 & 3 \\
\hline $\mathrm{K} 2$ & 0 & 61 & 71 & 13 & 22 & 2 & 1 & 12 & 182 \\
\hline K3 & 0 & 31 & 57 & 4 & 10 & 7 & 0 & 21 & 130 \\
\hline S1 & 40 & 1 & 0 & 0 & 1 & 0 & 0 & 0 & 42 \\
\hline S2 & 122 & 124 & 5 & 1 & 5 & 0 & 0 & 3 & 260 \\
\hline S3 & 9 & 16 & 0 & 0 & 2 & 0 & 0 & 0 & 27 \\
\hline \hline Total & 171 & 234 & 133 & 18 & 42 & 9 & 1 & 36 & 644 \\
\hline Fraction & 0.27 & 0.36 & 0.20 & 0.03 & 0.07 & 0.01 & $<0.01$ & 0.06 & 1.00 \\
\hline \hline
\end{tabular}

\subsubsection{Main formation channels}

Table 1 lists the main formation channels for CVs in our simulations. For comparison, we also included the corresponding formation channels described in Ivanova et al. (2006). Briefly, we have $\mathrm{CV}$ formation through CEP with/without weak/strong dynamical interactions, $\mathrm{CV}$ formation through exchanges with/without CEP, and CV formation through mergers with/without CEP. We are able to reproduce with MOCCA only some of the channels in Ivanova et al. (2006), since in MOCCA tidal capture (including collisions between red giants (RGs) and MS stars that lead to the formation of WD-MS binaries) is not modeled.

We define our formation channels as follows:

(1) CEP without any dynamical interactions, either before or after the CEP;

(2) CEP with weak/strong dynamical interactions, either before or after the CEP;

(3) exchange without any dynamical interactions post-exchange;

(4) exchange with dynamical interactions post-exchange;

(5) exchange followed by a CEP;

(6) merger without any dynamical interactions post-merger;

(7) merger with dynamical interactions post-merger;

(8) merger followed by a CEP post-merger.

\subsubsection{Comparisons with Ivanova et al. (2006)}

From the numbers in Table 1, we see that our results agree roughly with those found by Ivanova et al. (2006). Here, we discuss only a restricted number of models, by considering only those of our models with initial conditions that match those in Ivanova et al. (2006).

The models in Ivanova et al. (2006) with low densities yield results very close to our models K1 and S1. Ivanova et al. (2006) showed that most CVs in such low density clusters form through a CEP without any influence from dynamics. We also found this for our model S1. Model K1, on the other hand, is more difficult to compare. This is because it is a Kroupa model and, for the binary stellar evolution parameters adopted in this investigation, no CVs 
formed in $\mathrm{K} 1$ without any influence from dynamics (Belloni et al. 2016).

Additionally, our 'aggregated/average' cluster is similar to their Standard model (compare last row of Table 1 and the first row in their Table 2). We also found that only $\sim 27$ per cent of CVs are formed purely through a CEP without any contribution from dynamical interactions. The rest of the CVs were involved in some kind of interaction(s). The channels connected with exchanges and mergers are also quite similar in both models (our 'aggregated' and their Standard). However, there are two important differences that we must mention.

First, we cannot compare the influence of tidal capture and the physical collision of an MS star with an RG, because such a feature is not implemented in MOCCA. Secondly, we have an average enhancement with respect to formation channel 2, relative to their results. This could be associated with the fact that they only analyzed CV formation in GC cores, and that MOCCA's cluster models evolve in time, while theirs do not.

The following issues should be kept in mind for meaningful interpretations of our results.

(i) We have a restricted number of models/initial conditions. Our models do not match those in their study, with a few exceptions.

(ii) We have evolving clusters. Their models remain frozen in time. Consequently, they may lack some effects related to the cluster dynamical evolution.

(iii) We have CV formation throughout the cluster. They considered only $\mathrm{CV}$ formation inside the core.

(iv) We do not have information about the influence of tidal capture. Ivanova et al. (2006), however, have ruled out this mechanism as an efficient way to produce CVs in GCs.

(v) We use the CEP efficiency given by Hurley et al. (2002), i.e. $\alpha=3.0$, which might be inappropriate (Zorotovic et al. 2010). They use a CEP parametrization, such as $\alpha \lambda=1.0$, which also does not seem to be appropriate (e.g. Camacho et al. 2014; Zorotovic et al. 2010). This is because the common-envelope binding energy $\lambda$ has different values for different giants.

All of these effects should be kept in mind when performing comparisons between our study and that of Ivanova et al. (2006). In general, as already pointed out, a general and rough overall agreement is found between these two investigations. A more thorough comparison will be performed in a forthcoming paper, that will include a larger sample of MOCCA simulations.

\subsubsection{The role of exchange interactions}

Dynamics exchanges more massive CVs into the PDP. This was already noted by Ivanova et al. (e.g. 2006). Typically, a binary composed of low-mass MS stars will have one of them replaced by either a more massive MS star or a more massive WD. In the former case, the binary will eventually undergo a CEP, producing products with higher masses (at least for the WD mass). This happens in roughly 20 per cent of such exchange interactions. In 70 per cent of CVs formed due to exchanges, a low-mass MS star is replaced by a more massive WD and there is no CEP. Mass transfer starts after circularization and/or dynamical interactions and/or angular momentum loss. In the remaining 10 per cent, the pre-exchange binaries were composed of an RG and an MS star. In these cases, the MS star is replaced by another MS star with similar mass, followed by a CEP.

Ivanova et al. (2006) found similar results. They found that $\sim$ 20 per cent of the CVs formed post-exchange underwent a CEP (the last exchange led to an MS-MS binary), $\sim 80$ per cent of $\mathrm{CV}$ formed post-exchange did not pass through a CEP (the last exchange led to an MS-WD binary).

\subsubsection{Acceleration and Retardation of CV formation}

An additional effect associated with dynamical interactions is that they can either retard or accelerate CV formation. Shara \& Hurley (2006) noticed evidence for the cluster environment accelerating CV formation (e.g., their CV6). In this work, we confirm this possibility, but also the inverse, i.e. $\mathrm{CV}$ formation that is retarded due to dynamical interactions. We have only one case in which there is a strong dynamical interaction after CV formation, which led to an acceleration of the CV evolution. This case will be discussed in Section 3.5.2.

For accelerated $\mathrm{CV}$ formation, the $\mathrm{CV}$ progenitor interacts with single stars (or, more rarely, with softer binaries), producing a harder (i.e., smaller period) and/or a more eccentric binary. Both effects lead to a smaller pericentre distance. This causes the dynamically unstable mass transfer from the giant to take place earlier, when the giant is not so evolved (i.e., it has a small radius). This precedes a CEP, and in turn CV formation. Such CVs correspond to $\sim 30$ per cent of all $\mathrm{CVs}$ that had their formation times altered by dynamical interactions. Additionally, these CVs have smaller WD masses (since the increase of the giant core mass stops earlier) than their field-like counter parts.

For retarded $\mathrm{CV}$ formation, the $\mathrm{CV}$ progenitor interacts with harder binaries, causing it to become softened (i.e., migrating to longer periods). This effect leads to a greater pericentre distance, which makes dynamically unstable mass transfer from the giant take place later, when the giant is more evolved (i.e., it has a large radius). Thus, the $\mathrm{CV}$ progenitor starts the CEP later, which produces a more massive WD since the giant core has more time to evolve and increase its mass. Retardation takes place more frequently than acceleration, corresponding to $\sim 70$ per cent of all cases.

To sum up, the dynamical environment can either accelerate or retard $\mathrm{CV}$ formation due to dynamical interactions. Although Shara \& Hurley (2006) performed a systematic study of CV formation with NBODY6, their investigation suffered from small-number statistics (i.e., only two models). Nevertheless, the wealth of detail provided for the few CVs formed in their simulations makes it possible to compare their results to ours.

\subsection{The cluster evolution and the $\mathrm{CV}$ formation rate}

We show in this section that the $\mathrm{CV}$ formation rate can be divided into two distinct regimes: a burst in the beginning $(\lesssim 1 \mathrm{Gyr})$ followed by a nearly constant formation rate after $\sim 1$ Gyr, regardless of either the initial cluster properties (including the initial binary fraction, initial binary population, and initial central density), the cluster evolution or the strength of dynamical interactions during the formation process.

\subsubsection{Cluster Evolution}

In this section, we discuss the time evolution of the cluster properties in order to quantify the impact on $\mathrm{CV}$ formation.

Fig. 2a shows the behaviour of the core radius, calculated according to Casertano \& Hut (1985). Recall that the six models can be roughly described as: two sparse (K1 and $\mathrm{S} 1)$, two dense (K2 

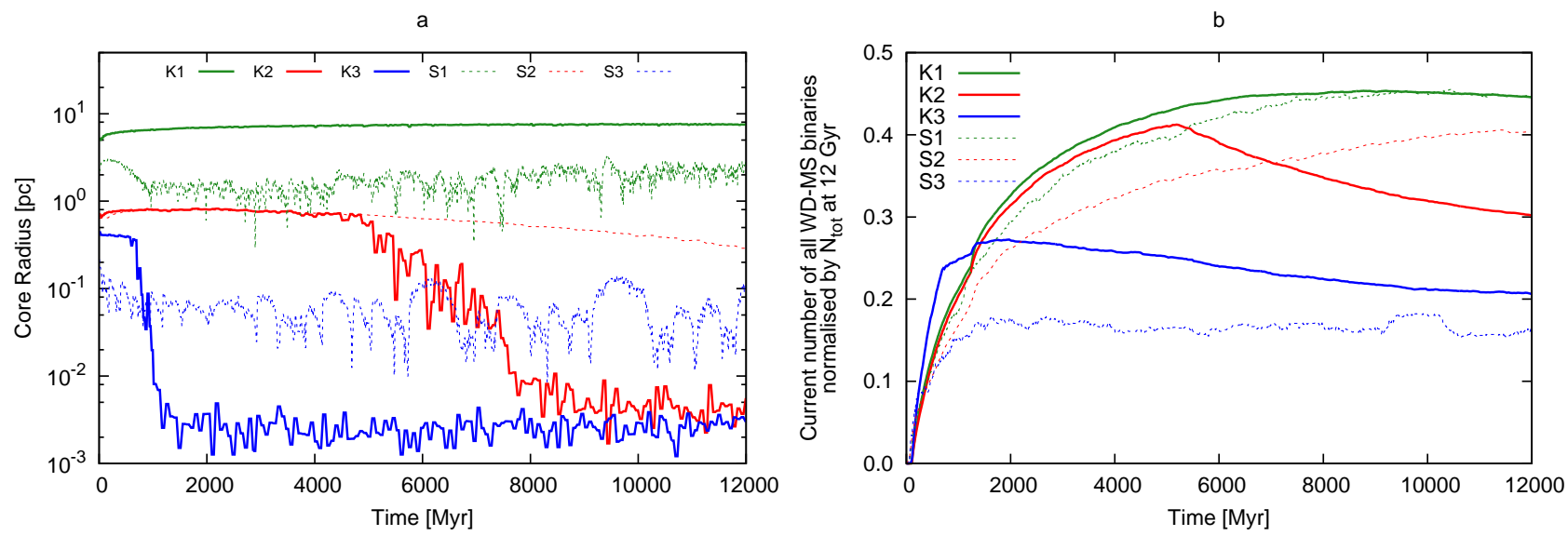

C
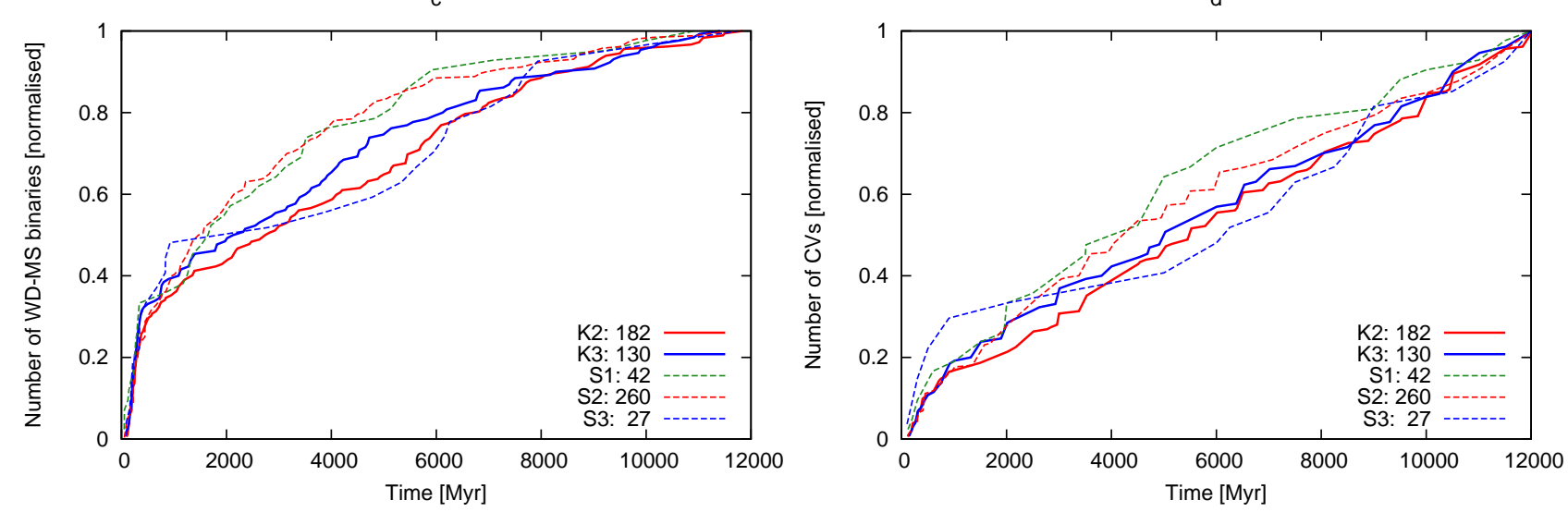

Figure 2. Top left: core radius $\left(r_{\mathrm{c}}\right)$ evolution, computed according to Casertano \& Hut (1985). Note that, at 12 Gyr, there are 4 post-core collapse clusters (K2, $\mathrm{K} 3, \mathrm{~S} 1$ and S3); two pre-collapse clusters (K1 and S2); and two clusters with intermediate-mass black holes (K2 and K3). Top right: number of detached WDMS binaries in the cluster at particular times, which corresponds to the total number of WD-MS binaries formed minus the total number of WD-MS binaries destroyed up to particular times, normalized with respect to the total number of WD-MS binaries formed up to 12 Gyr. Bottom left: cumulative number of WD-MS binaries that will at some point form the present-day CV population, normalized by the number of present-day CVs. Bottom right: cumulative number of present-day CVs, normalized by the number of present-day CVs. Notice that model K1 is not shown because it has only 3 present-day CVs. For details, see Section 3.3.

and S2) and two very dense (K3 and S3) models; four post-core collapse ( $\mathrm{S} 1, \mathrm{~S} 3, \mathrm{~K} 2$ and $\mathrm{K} 3$ ) models, and two with intermediatemass black holes (K2 and K3).

For model K1, the cluster is expanding and has a long relaxation time-scale. This is due to the cluster's large size, causing it to fill its tidal radius with an extremely low central density.

On the other hand, model S1 is comparably large, with a low density, and it experienced an episode of core-collapse around 1 Gyr. This model is particularly interesting because pre-corecollapse, it managed to form a black hole subsystem in the core. Breen \& Heggie (2013) explain the evolution of this kind of core collapse; the energy generated in the core is regulated by two-body relaxation throughout the entire system. In clusters with a centrally concentrated black hole subsystem (without a massive central black hole), the energy is generated by three-body encounters in the core. As the black holes escape from the cluster, it undergoes another episode of core-collapse. Once the balanced evolution is restored, the core of low-mass stars needs to be compact to allow for the formation and interaction of binaries composed of less massive stars. It is worth mentioning that the lifetime of the black hole subsystem is determined by the relaxation time-scale of the cluster, not by the dynamical time-scale of the black hole subsystem, which is much shorter. For more details on this particular scenario, see Wang et al. (2016).

We note that for models with intermediate-mass black holes (K2 and K3), the prescription given by Casertano \& Hut (1985) for the core radius begins to break down. This is because the mass of the core is significantly affected by the intermediate-mass black hole, negatively affecting the determination of the core radius. Again, the black holes provide a source of energy for clusters K2 and K3. Additionally, the intermediate-mass black holes in these models are formed in the way explained by Giersz et al. (2015). The intermediate-mass black hole in model $\mathrm{K} 2$ forms quite early ( $\lesssim 1 \mathrm{Gyr}$ ), whereas the one in model $\mathrm{K} 3$ forms at $\sim 5 \mathrm{Gyr}$.

Model S2 is approaching core-collapse at the present-day. The collapse will occur at $13 \mathrm{Gyr}$, such that the core density in this cluster is currently increasing, and quickly.

Finally, model S3 has initially only 300k stars, although it is extremely dense. This makes the relaxation time short, and corecollapse takes place very early on (several Myr after the start of the simulation). 

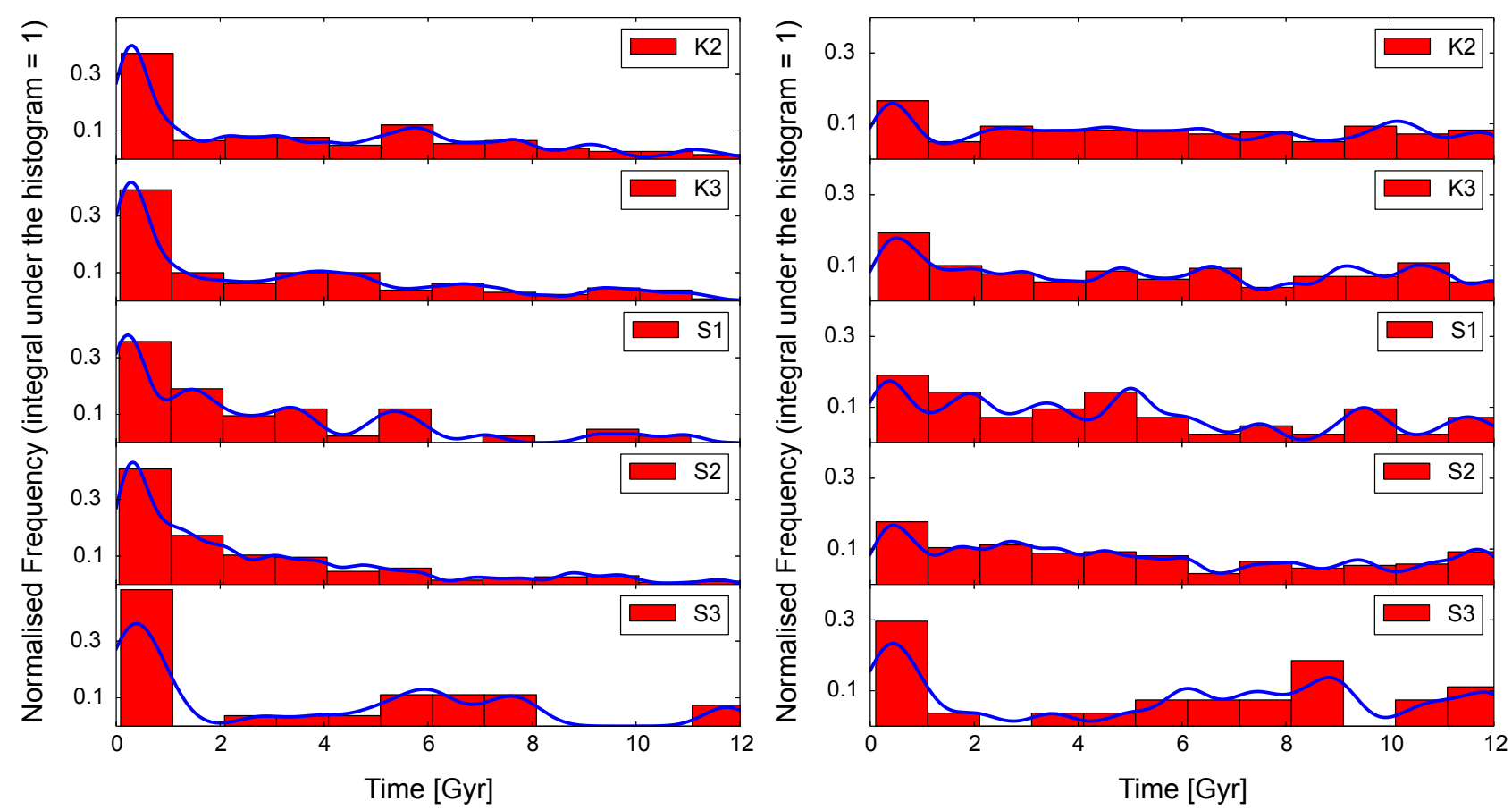

Figure 3. Left: formation rate $(\Delta N / \Delta t)$ of WD-MS binaries that will later become the present-day CV populations in five models. Right: formation rates $(\Delta N / \Delta t)$ of the present-day CVs in the five models. In both panels, the line corresponds to the probability density function estimated by the kernel density estimation method using Gaussian kernels with a bandwidth of $\sim 0.1$. Notice that model K1 is not shown because it has only 3 present-day CVs. Note also that the WD-MS binary formation rate and the CV formation rate show an initial burst, followed by smooth monotonic decrease. However, the CV formation rate is flatter (nearly constant), due to a time-delay between WD-MS binary formation and CV formation.

\subsubsection{Reservoir of WD-MS binaries}

Before discussing the $\mathrm{CV}$ formation rate, we stress that WDMS binary formation and CV formation are two distinct processes. WD-MS binary formation involves the formation of a detached binary composed of a WD and an MS star (due to a CEP, exchange, merger, etc.). $C V$ formation involves the formation of a semi-detached WD-MS binary, i.e. an interacting WD-MS binary. Both formation channels can occur at any time, a priori. For instance, a massive PCEB (formed in the first few Myr of the cluster evolution) can become a $\mathrm{CV}$ at any time, depending on its orbital period and on its MS mass, just after the CEP. If the period is small and the MS mass is great, then CV formation takes place fast. On the other hand, if the period is long and the MS mass is small, then the PCEB might have to wait a few Gyr (in order for its orbit to shrink due to angular momentum loss) until it initiates the $\mathrm{CV}$ phase.

We now move on to a brief analysis of the number of WD-MS binaries in clusters as a whole, i.e. let us first concentrate on all WD-MS binaries present in our simulated clusters throughout their evolution.

With respect to the formation of WD-MS binaries in the six models, two features are worth highlighting: an initial burst followed by a smoothly decreasing formation rate. This is due to the MS lifetime. Since the main-sequence lifetime is inversely related to mass, we see a very fast formation rate of WDs early in the cluster evolution (due to the fast evolution of massive MS stars), followed by a smoothly decreasing formation rate (due to the evolution of MS stars whose masses are below $\sim 2.0 \mathrm{M}_{\odot}$ and spend more time on the MS).

Fig. $2 b$ shows the number of WD-MS binaries in the clusters normalized by the total (cumulative) number of WD-MS binaries formed up to 12 Gyr. We find that models K1, S1, and S2 show a similar behaviour as described above, since the number of WD-MS binaries is continuously increasing. Hence, in these three models, this is reflected in the number of WD-MS binaries, which implies in turn that most WD-MS binaries formed in clusters are not destroyed (not strongly affected by dynamical interactions).

On the other hand, for models $\mathrm{K} 2$ and $\mathrm{K} 3$, we see that, at a particular time, the destruction of WD-MS binaries becomes more prominent. This is evidenced by the fact that the number of WD-MS binaries, from this point on, starts to decrease. Interestingly, the times at which the destruction process begins to dominate are precisely the times corresponding to the formation of their intermediate-mass black holes, in both models. This is consistent with the work of Leigh et al. (2014) and Giersz et al. (2015). The intermediate-mass black holes form at $\sim 1$ and $\sim 5 \mathrm{Gyr}$, for models $\mathrm{K} 2$ and $\mathrm{K} 3$, respectively.

Another interesting feature comes from comparing the K models collectively to the $\mathrm{S}$ models. Notice that the K curves in Fig. $2 \mathrm{~b}$ are always above the $\mathrm{S}$ curves, when the WD-MS binary formation rate surpasses the destruction rate, in all models. This is a consequence of the period distribution of the initial binaries. In the $\mathrm{S}$ models, the relative number of hard binaries is greater, in comparison with the $\mathrm{K}$ models (that have predominantly wide binaries). This makes mergers during or after a CEP more frequent for PCEBs in the $\mathrm{S}$ models. This way, even when the production rate dominates, many binaries merge on their way to becoming WD-MS binaries.

Finally, model S3 shows a weak destruction rate of WD-MS binaries up until $\sim 1$ Gyr. After that, we see clearly that the number 


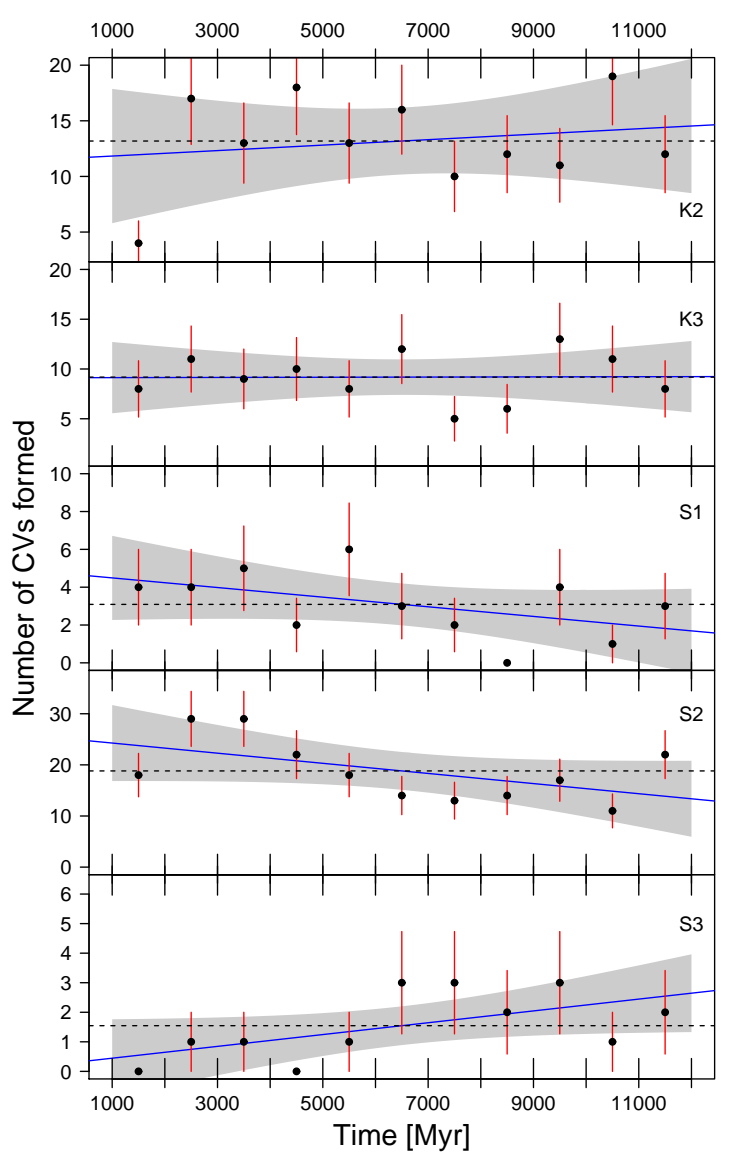

Figure 4. $\mathrm{CV}$ formation rate. Points represent the number of CVs formed after $1 \mathrm{Gyr}$, in intervals of $1 \mathrm{Gyr}$. The error bar represents Poisson errors $(\sqrt{n})$, and the blue line is the linear regression model $\left(N_{\mathrm{for}}=a+b t\right.$, where $N_{\text {for }}$ and $t$ are the number of CVs formed and the time, respectively). The gray area is the band of $95 \%$ confidence interval around the model. The dashed horizontal line is the average rate, assuming a constant $\mathrm{CV}$ formation rate (i.e. $b=0$ ). Note that all dashed lines are inside the gray bands, which indicates that all models are compatible with a constant formation rate. However, models S2 and S3 are in the extreme of acceptability, since their parameters $b$ are barely significant ( $p$-values 0.08 and 0.06 , respectively, but still not higher than the classical 0.05 for statistical significance).

of WD-MS binaries in the cluster remains roughly constant, which means that the WD-MS binary formation rate balances the destruction rate.

All of the aforementioned processes reflect on the rate at which WD-MS binaries become the present-day CV population. Additionally, we see clearly from Fig. $2 b$ that dynamics brings about a different evolution in the number of WD-MS binaries in each type of cluster, which provides a clue to the dynamical age of the cluster.

\subsubsection{Formation rate of WD-MS binaries that are CV progenitors}

Fig. 2c shows the cumulative number of WD-MS binaries that will later become the present-day CV population. We can separate the WD-MS binary formation rate into two distinct regimes: an initial burst that lasts up to $\sim 1 \mathrm{Gyr}$, followed by a roughly smoothly decreasing formation rate. This is analogous to the formation rate of the WD-MS binary populations in our simulated clusters. This is apparent from the left-hand panel of Fig. 3, which shows this formation rate.

We also find that models $\mathrm{S} 1$ and $\mathrm{S} 2$ show a more prominent decrease in the $\mathrm{CV}$ progenitor formation rate relative to models $\mathrm{K} 1$ and K2 (compare first/second and third/fourth rows of Fig. 3). This is associated with the cluster density, and it reflects the intimate balance between formation and destruction. Models S1 and S2 are less dense than models $\mathrm{K} 2$ and $\mathrm{K} 3$. This makes the destruction of potential CV progenitors less frequent in models $\mathrm{S} 1$ and $\mathrm{S} 2$. These two different behaviours (one for models S1 and S2, and the other for models K2 and K3) are intrinsically associated with the influence of dynamics. In models $\mathrm{S} 1$ and $\mathrm{S} 2$, the production of WD-MS binaries is driven by stellar evolution processes. On the other hand, models $\mathrm{K} 2$ and $\mathrm{K} 3$ are much denser and are post-core collapse clusters, which increases the dynamical production rate of WD-MS binaries. Nevertheless, we see a general pattern associated with an initial burst in the formation rate, followed by a roughly smoothly decreasing rate. The intensity of such a decrease depends on the main $\mathrm{CV}$ formation channel. For CVs associated with CEP (with/without weak dynamical interactions), like those predominantly formed in models S1 and S2, we note a more rapid decrease in the formation rate in the first Gyr. On the other hand, for dynamically formed CVs (models K2 and K3), we see a less rapid decrease (compare KDE models in Fig. 3). Then, dynamics tend to 'flatten' the WD-MS formation rate.

Finally, model S3 shows a combination of the above two features, since this model is extremely dense. This leads to the presence of a significant population of dynamically-formed CVs. Meanwhile, CVs also form purely through stellar and binary evolution. We see the initial burst of WD-MS binary formation, followed by a peculiar time-dependence for the formation rate (see the bottom row of Fig. 3). Unfortunately, we are not able to say more about this model, since its number of CVs is very small.

\subsubsection{CV formation rate}

Having explained the WD-MS binary formation rate, we now turn to the $\mathrm{CV}$ formation rate. Once a WD-MS binary forms, there is a time-delay before CV formation. The duration of this delay depends on the period and MS mass of the WD-MS binary.

Comparing Figs. $2 \mathrm{c}$ and $2 \mathrm{~d}$, we see that the cumulative numbers of WD-MS binaries and CVs are different. This difference is due to the above-mentioned time-delay between WD-MS binary and $\mathrm{CV}$ formation.

In any event, from Fig. $2 \mathrm{~d}$ and the right-hand panel of Fig. 3 (which shows the CV formation rate), we see again an initial burst. However, for the CVs, a nearly constant formation rate follows the initial burst (different from the WD-MS binary formation rate, that shows a roughly smoothly decreasing formation rate following the initial burst). This was already noticed by Ivanova et al. (2006), who concluded that the cluster evolution is not important with respect to the relative number of CVs appearing in GCs.

In order to test this claim, we applied a two-sample Kolmogorov-Smirnov test to pairwise combinations of all five models. Since the alternative hypothesis of the test is that the paired samples do not stem from the same theoretical CV formation rate, a null result supports the conclusion that the $\mathrm{CV}$ formation rate is model-independent, in the sense that the $\mathrm{CV}$ populations form in a similar way irrespective of the cluster properties.

None of these tests support the rejection of the null hypoth- 
esis with $99 \%$ confidence, which suggests that there is no strong evidence that the $\mathrm{CV}$ formation rate differs from model to model.

However, these results are sensitive to small number statistics. In fact, when models $\mathrm{K} 2$ and S2 are tested, we can reject the null hypothesis that they are similar with a confidence level greater than $97 \%$ ( $p$-value $=0.02$ ). For these two models, the KS test suggests that we have enough evidence to reject the null hypothesis. This is important, since these two models have the largest numbers of CVs. It is likely that the uniqueness of the $\mathrm{CV}$ formation rate amongst different GC models can only be correctly analyzed with larger simulations yielding more $\mathrm{CVs}$.

In order to test whether the CV formation rate is indeed nearly constant after $\sim 1 \mathrm{Gyr}$, we applied a one-sample KolmogorovSmirnov test for uniformity to the same five models. Only model S2 presents a small $p$-value $\left(1.224 \times 10^{-4}\right)$. This is because more CVs form in model $\mathrm{S} 2$, and it ends up being easier to identify deviations in this model, i.e. it is easier to notice that model S2 does not follow the other models. The other models do seem different, but the small number statistics make them technically indistinguishable.

Proceeding further, we performed a linear regression for the number of CVs formed after $1 \mathrm{Gyr}$, in each interval of $1 \mathrm{Gyr}$, using the absolute numbers in Fig. 3. If the CV formation rate is constant, then a fit of the form $N_{\text {for }}=a+b t$, where $N_{\text {for }}$ is the number of $\mathrm{CVs}$ formed and $t$ is the time, should return a value for $b$ that is consistent with zero (i.e. the error is much greater than the value of the coefficient itself) For all models, only S2 and S3 have barely significant coefficients $b$, albeit marginally ( $p$-value $=0.08$ and 0.06 , for S2 and S3, respectively), reinforcing the uniformity test indicated above. The $\mathrm{CV}$ formation rate in model $\mathrm{S} 2$ is not exactly constant. The other models are compatible with the hypothesis of a constant rate.

The result of this last test is shown in Fig. 4, which shows the number of CVs formed in intervals of $1 \mathrm{Gyr}$. In this figure, the blue line is the linear regression, the gray band is the $95 \%$ confidence interval around the linear regression (which indicates the error in the fitting procedure), the error bars correspond to Poisson errors $(\sqrt{n})$, and the dashed line is the average rate (assuming a constant rate, i.e. $b=0$ ). Note that straight lines within the gray band are compatible with the data, to within the fitting errors. Additionally, all dashed lines (average rates) are inside the gray band, which indicates that a constant $\mathrm{CV}$ formation rate is coherent for all models, although models S2 and S3 barely fit this criterion, since their b values are barely non-zero ( $p$-values of 0.08 and 0.06 , respectively, but still higher than the classical 0.05 for statistical significance).

We emphasize that the one-sample KS test and the linear regression fitting are different procedures, which leads to different results. The former should be somewhat more precise, since the latter depends on the choice of binning for the histogram counts. However, given the differences in the results, it seems reasonable to assume that the $\mathrm{CV}$ formation rate is nearly constant.

To summarize, the WD-MS binary formation rate shows an initial burst, followed by a roughly smoothly decreasing rate. The $\mathrm{CV}$ formation rate, on the other hand, has an initial burst, followed by a nearly constant formation rate. This difference is due to the time-delay between WD-MS binary and CV formation. This suggests that we should expect the same CV formation rate in all types of clusters. However, only by analyzing more models can we better define any dependence of the WD-MS binary and CV formation rates on the host cluster properties and evolution.

\subsubsection{Comparisons with Ivanova et al. (2006)}

Now that the dependences of the WD-MS binary and CV formation rates on the cluster properties have been described, we compare our general picture with the findings of Ivanova et al. (2006).

In their Fig. 8, they show the rate of occurrence of the last major dynamical event or CEP. Notice that this rate corresponds closely to our WD-MS binary formation rate. They show that the occurrence of CEPs is mostly within the first $\sim 3$ Gyr of cluster evolution, and the occurrence of dynamical events is distributed almost uniformly in time.

As discussed in Section 3.3.4, we find in our simulations an enhancement in the formation rate of WD-MS binaries in the first few Myr of the cluster evolution, for both PCEBs and dynamically formed WD-MS binaries. This is followed by a smoothly decreasing rate. Both episodes can be explained by the evolution in the turn-off mass, and the interplay between formation and destruction of WD-MS binaries.

The above differences in our findings can be explained by the differences inherent to both approaches. In our case, we model CV formation throughout the entire cluster, whereas they consider only formation in the core. Additionally, their clusters are frozen in time, while ours evolve dynamically. As a result, they might be missing some of the CVs formed in our simulations, either at early or late times, which are of course responsible for the above-mentioned features.

In their Fig. 9, they show the initial appearance of CVs (i.e., the onset of mass transfer) in their simulations. This corresponds to our CV formation rate. They find for CVs formed from a CEP that the $\mathrm{CV}$ formation rate is roughly constant in time. For dynamically formed CVs, they find an increase in the formation rate after $\sim 7$ Gyr. In our simulations, we find that CVs formed through all channels show an enhancement in the rates at the beginning of the cluster evolution, followed by a nearly constant rate after $\sim 1 \mathrm{Gyr}$. This is accounted for by the time-delay between WD-MS binary formation and CV formation. Additionally, we applied a two-sample KS test, and concluded that we do not have enough evidence to reject with 99 per cent confidence (or more) the null hypothesis that the $\mathrm{CV}$ formation rate is unique for all GCs.

In general, the overall results of the two studies corroborate, especially given their respective limitations. Yet to come detailed investigations could define with more confidence the relation between the cluster properties and the $\mathrm{CV}$ formation rate.

\subsection{The formation-age population}

The WD and donor mass, and period distributions of the CVs at the moment they are formed are shown in Fig. 5. In what follows, we focus more on the bottom row of each panel, which corresponds to our 'aggregated/average' cluster.

\subsubsection{Primary Mass}

We see from the WD mass distribution (5a) that there is a clear separation between dynamically formed CVs and CVs formed without any influence from dynamics. For the CVs in the BSE group, the peak is $\lesssim 0.25 \mathrm{M}_{\odot}$, which means that those $\mathrm{CV}$ s formed from CEPs without any influence from dynamics typically have $\mathrm{He}$ WDs rather than C/O WDs. Additionally, there is a smaller peak at $\sim 0.7 \mathrm{M}_{\odot}$, which is due to massive WDs formed early on in the cluster evolution.

On the other hand, CVs in the SDI group have more massive 
a

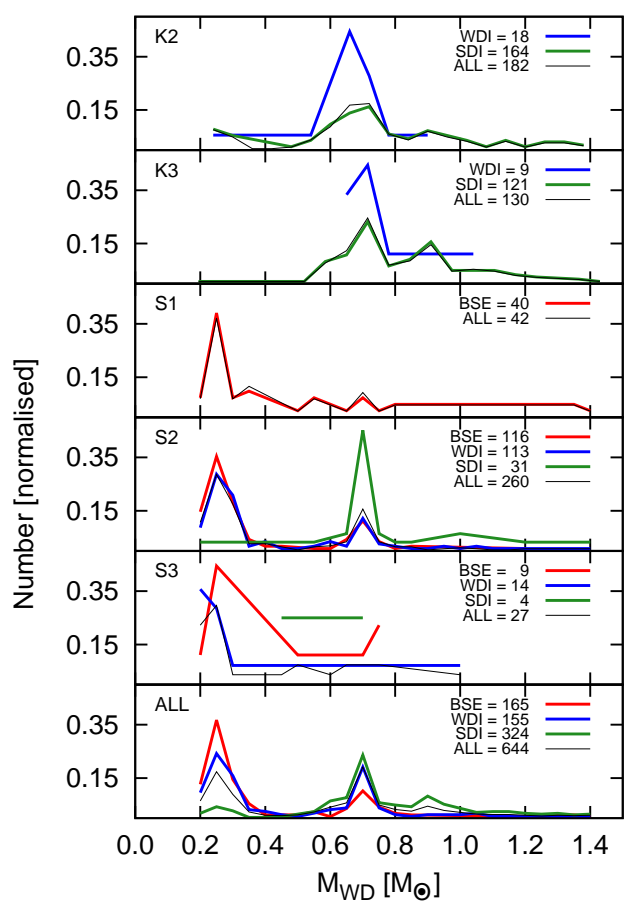

b

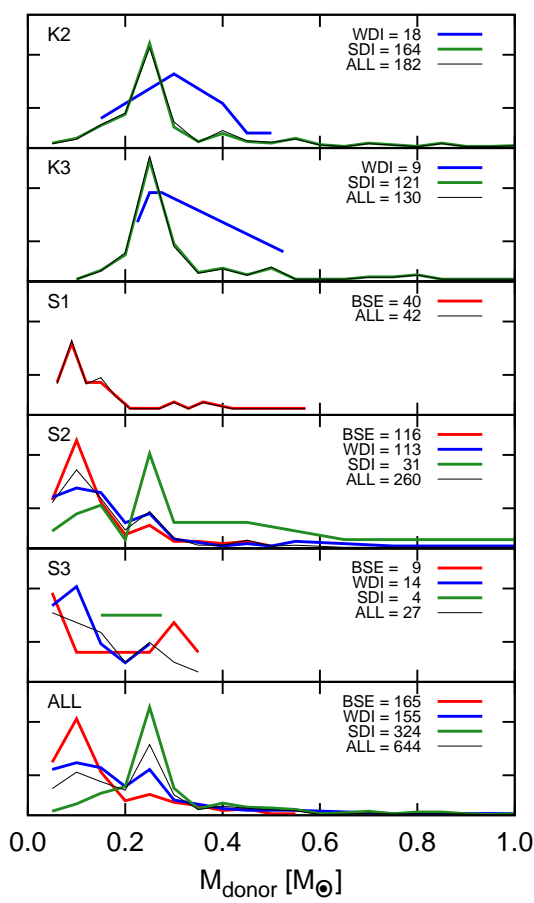

C

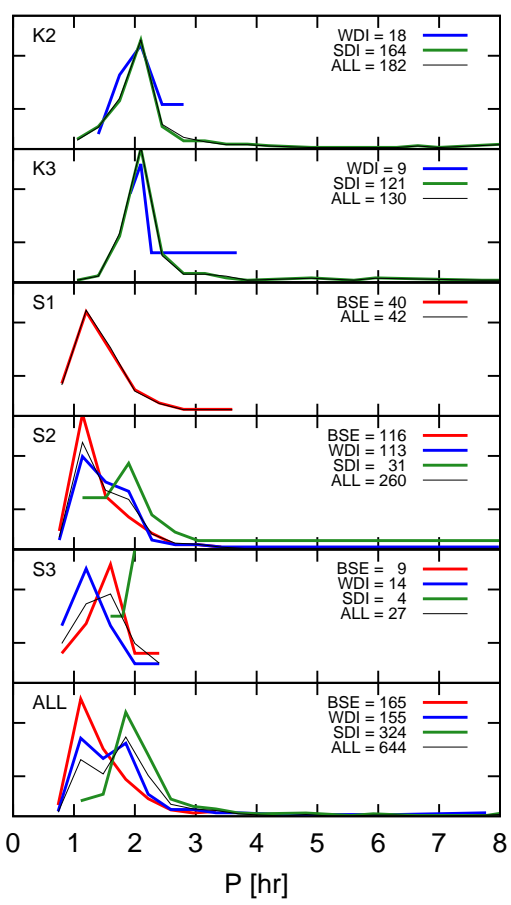

Figure 5. WD mass (left-hand panel), donor mass (middle panel) and period (right-hand panel) distributions associated with CVs that survive up to the present-day, shown at their formation times in the six models. Keys and the bottom row are similar, as in Fig. 1. We can see a clear distinction between CVs formed under the influence of strong dynamical interactions (SDI group) and CVs formed without any influence from dynamical interactions (BSE group). CVs in the BSE group have predominantly low-mass WDs and donors, and extremely-short periods. CVs in the SDI group have higher WD and donor masses, and longer periods. CVs formed via the weak influence of dynamics (WDI group) have properties similar to CVs in the BSE and SDI groups. For more details, see Section 3.4.

WDs, with a peak at around $0.7 \mathrm{M}_{\odot}$. This is explained via the role of exchanges in the $\mathrm{CV}$ formation process (Section 3.2.3). There are other small peaks in the distribution as well, at $\sim 0.25$ (caused by the evolution of low-mass MS stars) and $\sim 0.9 \mathrm{M}_{\odot}$ (caused by the evolution of high-mass MS stars).

$\mathrm{CVs}$ in the WDI group have roughly the same relative numbers of massive and light WDs. This is due to the net effect of weak dynamical interactions. If the net effect is great, then more massive systems can be bumped into the required range in parameter space to form CVs. On the other hand, if the net effect is weak, then these interactions are unable to change drastically the binary properties, such that the CV will have similar properties to those found in the BSE group.

CVs in the WDI group in the Kroupa models are similar to those in the SDI group. This indicates that in these clusters, the net effect of weak dynamical interactions is strong. For the Standard models, most CVs in the WDI group behave analogously to those in the BSE group (the net effect of weak dynamical interactions is weak). However, we see a few for which the cumulative effect is strong. These objects have similar features as the CVs in the SDI group.

Importantly, the WD mass distribution at the time of formation is very similar to that at the present-day. This is because BSE assumes that only 0.001 per cent of the accreted matter is, in fact, accreted by the WD (Hurley et al. 2002, Eq. 66). Considering the low mass transfer rates of these systems (short-period CVs), we should not expect too much of a difference in the WD masses. This is a reasonable expectation since, due to nova eruptions, most of the accreted material is indeed expelled (but not all).

\subsubsection{Secondary Mass}

As for the donor mass, we see the same behaviour for the WD mass, i.e. a clear separation between dynamically formed CVs and $\mathrm{CVs}$ formed without any influence from dynamics. Dynamically formed CVs have more massive donors at the formation time, relative to CVs not influenced strongly by dynamics, which is in good agreement with the findings in Shara \& Hurley (2006).

This is once again associated with exchanges. From the second panel of Fig. 1, we see that most $\mathrm{CV}$ progenitors in the SDI group have mass ratios greater than $\sim 0.2$. This is the limit for $\mathrm{CV}$ formation through pure stellar evolution (Section 3.1.3). These binaries have to have at least one binary component replaced in order to become CVs. After the exchange, the resulting binaries are composed of a massive star (either a WD or an MS star) and an MS star with mass $\lesssim 0.5 \mathrm{M}_{\odot}$. These MS stars are future $\mathrm{CV}$ donors.

On the other hand, CVs in the BSE group have donor masses $\lesssim 0.2 \mathrm{M}_{\odot}$, because their progenitors had such a small range of masses (see mass ratio and primary mass distributions in Fig. 1).

It is important to note that we do not find any difference in the donor mass in the BSE group relative to what is found for the dynamics groups at 12 Gyr (Belloni et al. 2016, see their Fig. 2). This is the result of CV evolution (mass loss).

In the end, most $\mathrm{CV}$ donors have masses $\lesssim 0.35 \mathrm{M}_{\odot}$, which implies that they are born either as short-period CVs or as gap CVs. 


\subsubsection{Mass Ratio and Period}

Beginning with the mass ratio distribution, almost all CVs have mass ratios less than 1 (only one $\mathrm{CV}$ in model $\mathrm{S} 2$ has $\mathrm{q}>$ 1). This means that they are thermally stable. However, some of them could be dynamically unstable (see Section 3.4.4). Additionally, most CVs have mass ratios between 0.2 and 0.6 , and there is no clear distinction among the BSE, WDI and SDI groups.

As already inferred from the donor masses, most CVs have periods $\lesssim 3 \mathrm{~h}$, which means that they are short-period CVs or gap $\mathrm{CV}$. We found in our simulations that the gap is apparently missing.

In general, dynamically formed $\mathrm{CV}$ s have longer periods, since they have heavier donors. CVs in the BSE group have very low mass donors, which leads them to have very short periods. This comes from the fact that CV donors fill their Roche lobes, which produces a rough relation between donor mass and $\mathrm{CV}$ period (e.g. Knigge 2011, see section 2.1).

\subsubsection{Discussion on the Formation-Age Population}

We begin this section with a few comments regarding potential biases that could affect any interpretations of our results. First, with respect to WD mass, it might be that most of the low-mass CVs (composed of He WDs) are dynamically unstable. According to Schreiber et al. (2016), if the strength of consequential angular momentum loss is inversely proportional to the WD mass, then most CVs with WD masses less than $\sim 0.5 \mathrm{M}_{\odot}$ are dynamically unstable. Note that we use the term "consequential angular momentum loss" to refer to AML that is a consequence of mass-loss. The motivation for such a functional form comes from the fact that a He WD has never been observed in a CV in the Galactic field, although such WDs are observed in Galactic detached systems (Zorotovic et al. 2011). The primary mechanism thought to be responsible for such a dependence for the WD mass is nova eruptions (Nelemans et al. 2016; Schreiber et al. 2016). The frictional angular momentum loss produced by novae depends strongly on the expansion velocity of the ejecta (Schenker et al. 1998). For low-mass WDs, the expansion velocity is small (Yaron et al. 2005), and this leads to a strong angular momentum loss by friction. This, in turn, makes CVs dynamically unstable.

We emphasize that the apparent absence of the period gap in our simulations might be an intrinsic issue in the BSE code. This is because, even without dynamics, we cannot reproduce the period gap during the evolution of long-period CVs with BSE. This is most likely connected with the remaining improper implementations in BSE (e.g. tides and spins).

\subsection{From the formation-age to the present-day}

In this section, we briefly discuss $\mathrm{CV}$ evolution from the time of their birth until a cluster age of $12 \mathrm{Gyr}$. Special attention is given to one unique case that suffered a strong dynamical interaction after $\mathrm{CV}$ formation. As a general characteristic, $\mathrm{CV}$ s are hard enough to avoid any kind of strong encounter. However, some can still undergo weak interactions, which causes a minuscule change in eccentricity (see Leigh et al. (2016) for more details regarding the interruption of binary mass transfer due to dynamical interactions).

\subsubsection{CV evolution without dynamics}

For the most part, $\mathrm{CV}$ evolution without dynamics is as we would expect. That is, a CV evolves toward shorter periods up to the moment its donor changes its structure, and becomes an H-rich degenerate object. At this point, the $\mathrm{CV}$ begins to evolve toward longer periods.

As already mentioned, BSE cannot reproduce the period gap. This is apparent upon analyzing $\mathrm{CV}$ evolution for the long-period CVs. In BSE, there is a decrease in the rate of angular momentum loss when the donor reaches a mass of $\sim 0.35 \mathrm{M}_{\odot}$ (Hurley et al. 2002, section 2.4), because magnetic braking ceases to act. At this point, in reality, the donor would have time to restore thermal equilibrium and stop overfilling its Roche lobe. However, in BSE, tides (in combination with spins) keep the binary locked into mass transfer, which causes the period to increase in response.

From a statistical point of view, the aforementioned issues should not drastically affect our results. This is because our main objective in this paper is not to model particular CVs, but rather to establish an overall dynamical picture for $\mathrm{CV}$ formation and evolution in globular clusters.

\subsubsection{CV evolution with dynamics}

Next, we discuss CV evolution altered by some kind of dynamical interaction. Shara \& Hurley (2006) described one such case found in their simulations. This CV had its evolution accelerated due to dynamical interactions.

In our simulations, we found only one similar case (out of 644 $\mathrm{CVs}$ ), in model $\mathrm{K} 2$. Unfortunately, model $\mathrm{K} 2$ has only dynamically formed $\mathrm{CVs}$, so that we cannot make a comparison with a field-like CV population, as done by Shara \& Hurley (2006).

This $\mathrm{CV}$ in model $\mathrm{K} 2$ is not the result of an exchange. Instead, it is the result of a sequence of dynamical interactions that decreased its progenitor's orbital period, causing the outcome of the CEP to be a short-period binary. The CV formed at $\sim 200 \mathrm{Myr}$. After its formation, it was unaffected by dynamical interactions, up to $\sim 10 \mathrm{Gyr}$, at which point this $\mathrm{CV}$ interacted with a WD-WD binary. At this moment, the $\mathrm{CV}$ is already far past period bounce, and has a donor mass of $\sim 0.04 \mathrm{M}_{\odot}$ and a period of $\sim 3 \mathrm{~h}$. The WDWD binary initially had a period of $\sim 600 \mathrm{hr}$. After the interaction, as expected, the CV became harder, and the WD-WD binary softer. This hardening led to a $\mathrm{CV}$ with an orbital period of $\sim 1 \mathrm{~h}$, causing an acceleration in its subsequent evolution due to an enhancement in the rate of mass loss, and a decrease in donor mass (to a value $0.025 \mathrm{M}_{\odot}$ ). Within a fraction of a Myr after the interaction, the $\mathrm{CV}$ established a stable mass transfer rate with a period of $\sim 4 \mathrm{~h}$.

Even though both CVs (in our simulations and in their simulations) corroborate with respect to the pattern, we expect this behaviour to be rare in GCs (Leigh et al. 2016). This is because CVs are very dynamically hard, and of low mass, such that the impact parameter for interactions is small. It follows that the probability of an interaction occurring is very small. Only one case in our investigation and one case in the Shara \& Hurley (2006) study were found, suggesting that overall dynamics should only very rarely directly affect $\mathrm{CV}$ evolution.

On the other hand, as discussed in Section 3.2.4, pre-CVs are more likely to interact. Consequently, this is a more likely channel to eventually affect $\mathrm{CV}$ evolution, whether it be in the form of an acceleration or a retardation. But we emphasize that the influence of dynamics typically affects CV progenitors, not CVs directly. Dynamics acts indirectly, by affecting the progenitors. 


\subsection{Dependence on initial binary fraction}

In this section, we test to what degree the results presented in this paper (relative to Belloni et al. (2016)) depend on our assumptions for the initial binary fraction, for both adopted IBPs (high for Kroupa IBP and low for Standard IBP). To this end, we ran 3 additional models with the same initial cluster properties as in the $\mathrm{S} 1$, $\mathrm{S} 2$ and S3 models, but with an initial binary fraction of 95 per cent.

For these runs, models S1, S2 and S3 have semi-major axis distributions extending initially up to $50 \mathrm{AU}$. We emphasize that by increasing the initial binary fraction, we must also increase this maximum semi-major axis. Otherwise, the energy generated via single-binary encounters catalyzed by the presence of such a large population of very hard binaries would be incredibly high, and present-day clusters would have much higher binary fractions than observed. Consequently, in the Standard models with initial binary fractions of 95 per cent, the initial semi-major axis distribution extends to $10^{4} \mathrm{AU}$. After 10-12 Gyr of evolution, however, the cluster binary fraction (including all binary masses) has been reduced to around 30,20 and 10 per cent, in order of increasing cluster density. Most of these binaries are of low-mass, remain in the outskirts of the system, and do not affect the rate of energy generation due to binary interactions or the overall cluster evolution.

The resulting CV properties characteristic of these models are similar to models S1, S2 and S3. The main difference between these sets of models is the total number of CVs that are formed. Models with high initial binary fractions form more CVs than models with low initial binary fractions. But their CVs still form at an approximately constant rate due mainly to binary stellar evolution, are composed of mostly low-mass WDs, tend to form with short periods $(<2 h)$, and are often period bouncers at the present-day.

\subsection{CV properties and dependences on their age}

Having described the main features characteristic of the present-day CVs in our six models, from the time of cluster birth up to an age of $12 \mathrm{Gyr}$, we now turn our attention to a more general issue concerning the time evolution of the properties of $\mathrm{CV}$ populations, before addressing $\mathrm{CV}$ s that do not survive until the present-day (Section 3.8).

One important property of CVs, in the Galactic field or in GCs, is that $\mathrm{CV}$ populations as a whole change properties as they age. This is important for comparing field and cluster CVs, since cluster CVs might be up to 4 times older than field CVs.

Note that CVs are created continuously in time (see Fig. 2d). Hence, the properties of $\mathrm{CV}$ populations should change in time, due both to $\mathrm{CV}$ evolution and the constant addition of new $\mathrm{CV}$ s to the population.

In what follows, we describe the main changes in the CV population properties as a function of time. We concentrate on changes in the WD (Fig. 6) and donor mass (Fig. 7) distributions. In both figures, each box corresponds to the region between the first and third quartiles, with a horizontal line at the median value. Hence, 25 per cent of the data belongs to the region below the lower edge of the box, 25 per cent of the data belongs to the region above the upper edge of the box, and the box corresponds to 50 per cent of the data (interquartile region). Above and below the boxes there are two vertical lines, called the whiskers. The line above the box ends at a value which is 1.5 times the interquartile range (i.e. the vertical height of the box) from the upper edge of the box. Similarly, the line below the box ends at a value which is 1.5 times the interquartile range, from the lower edge. Each whisker is trun- cated at a value in mass belonging to a particular data point. Since it can occur that no data point has a value of exactly 1.5 times the interquartile distance, the whisker may be shorter than its nominal range. Additionally, a cloud of random points is shown to the left of each box, which corresponds to the underlying distribution.

In Fig. 6 and Fig. 7, two clusters were considered, namely S2 and $\mathrm{K} 2$. In order to perform comparisons to a field-like population, we added an additional population similar to model S2, but evolved using BSE alone (i.e. without dynamics). This third model is named in the figures as S-BSE. Importantly, the other $\mathrm{K}$ and $\mathrm{S}$ clusters show similar features. This is the reason we show only these two clusters in the figures.

\subsubsection{WD Mass}

Fig. 6 shows the time evolution of the WD mass distribution for CVs. Note that for model K2 (having only dynamically formed $\mathrm{CVs})$, the WD median mass stays roughly constant in time $(\sim 0.7$ $\left.\mathrm{M}_{\odot}\right)$. This illustrates what has already been said about the role of exchanges (Section 3.2.3), which put more high-mass WDs into the $\mathrm{CV}$ population. We also see a second inconspicuous peak for lowmass WDs around $0.3 \mathrm{M}_{\odot}$, which originates from $\mathrm{CVs}$ affected by weak dynamical interactions, and becomes more pronounced at $\sim$ 8 Gyr. Nevertheless, it is clear from the figure that model K2 is dominated by high-mass WDs. This is not true for model $\mathrm{S} 2$.

In models S2 (cluster CVs) and S-BSE (field-like CVs), we see a clear drop in the WD median mass, starting at $\sim 3 \mathrm{Gyr}$. This is caused by the continuous addition of low-mass WDs in to the CV population, due to the evolution of MS stars whose masses are $\lesssim 2$ $\mathrm{M}_{\odot}$. In the beginning (up to $\sim 1 \mathrm{Gyr}$ ), both models are dominated by high-mass WDs. After that, we see the addition of more lowmass WDs than high-mass WDs. We also see that the cluster CVs (model S2) have slightly more high-mass WDs than their field-like counterparts (S-BSE) throughout the evolution, due to a few CVs that are formed due to strong dynamical interactions. Furthermore, from the distributions near the boxes, we see that for these two models, the WD mass distribution becomes bimodal at $\sim 3 \mathrm{Gyr}$, although the peak for high-mass WDs $\left(\sim 0.7 \mathrm{M}_{\odot}\right)$ is much less pronounced than the peak for low-mass WDs $\left(\sim 0.3 \mathrm{M}_{\odot}\right)$.

Finally, we point out two main features in Fig. 6; (i) one associated with the time evolution of the WD mass distribution, and (ii) the other with the differences among the models.

(i) We expect a clear drop in the median WD mass over time due to the addition of low-mass WDs. However, if the cluster is dominated by dynamically formed CVs, we expect roughly no time evolution of the median value.

(ii) We observe an extension of the interquartile range in the early evolution for all models. However, for models S2 and S-BSE, a decrease is seen instead during the late-time evolution, which is caused by the predominance of low-mass WDs.

\subsubsection{Donor Mass}

From Fig. 7, we see a drop in the median value of the donor mass distribution for all models as time goes on. However, the drop is less severe for dynamically formed CVs (model K2). This indicates that $\mathrm{CV}$ populations in all models tend toward having extremely-low-mass donors at late times, regardless of the influence of dynamics or the formation channel. More specifically, dynamically formed CVs tend to have more high-mass donors, relative to $\mathrm{CV}$ s formed without any influence from dynamics (compare 

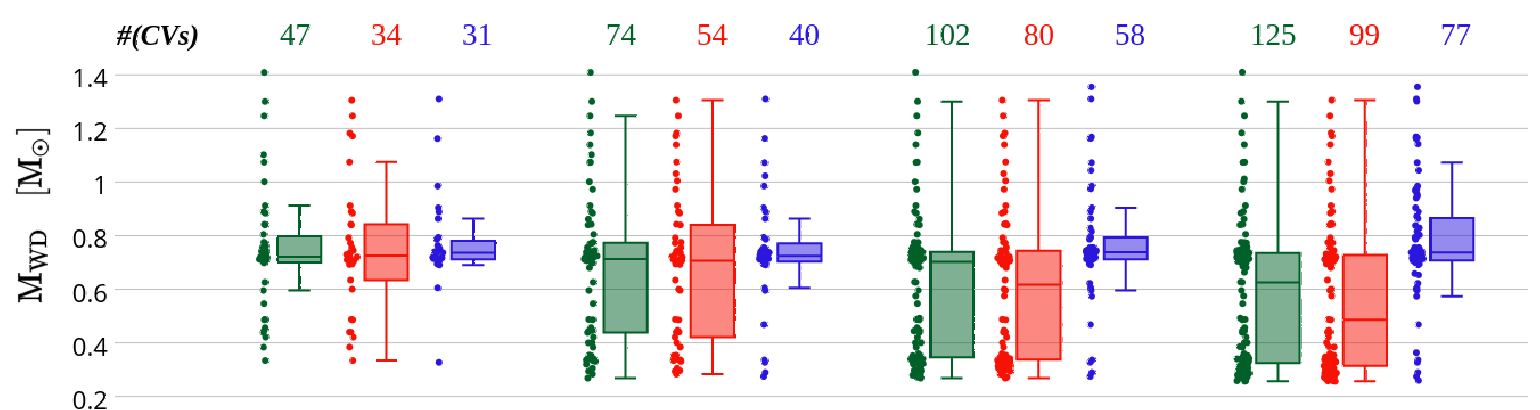

$\square \mathrm{S} 2$

$\square$ S-BSE

$\square \mathrm{K} 2$
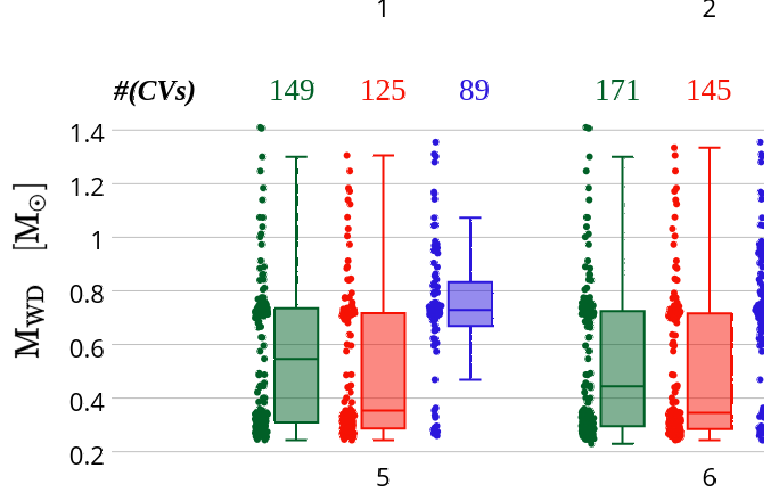

\section{Gyr}
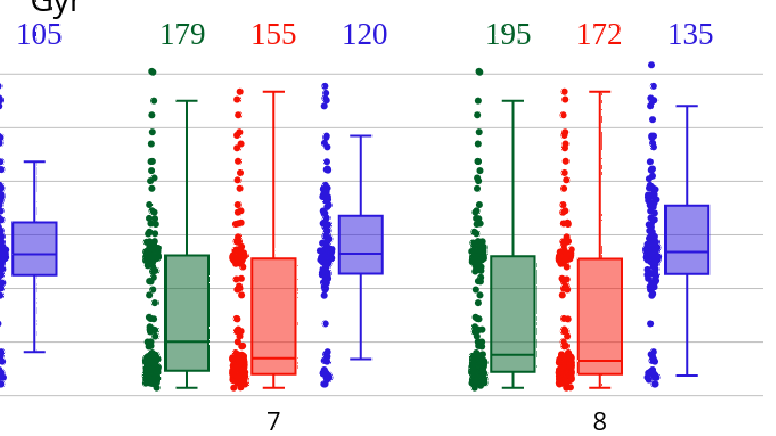

$\square \mathrm{S} 2$

$\square \mathrm{S}-\mathrm{BSE}$

$\square \mathrm{K} 2$
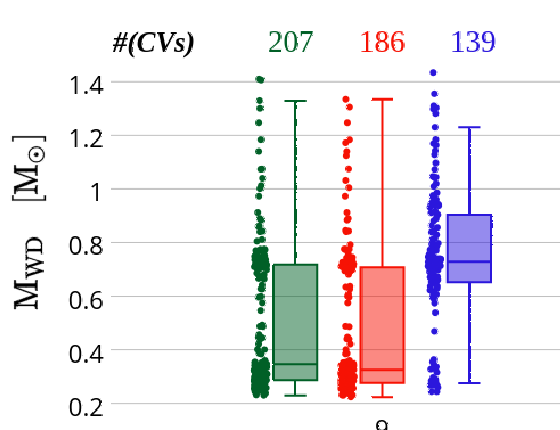

9)

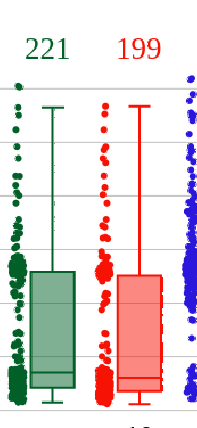

Gyr

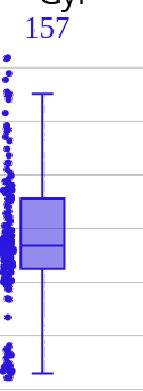

10

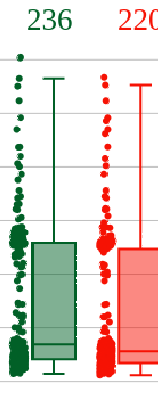

11

Gyr

Figure 6. Evolution of the WD mass distribution in time for all CVs in 2 out of the 6 models. Models K2 and S2 show the results for CVs evolved by MOCCA (i.e. they are associated with a cluster environment). Model S-BSE is related to model S2, but evolved using BSE alone (i.e. it is related to a field-like environment, without dynamics). Each box corresponds to the region between the first and third quartiles, with a horizontal line at the median value. Hence, 25 per cent of the data belongs to the region below the lower edge of the box, 25 per cent of the data belongs to the region above the upper edge of the box, and the box corresponds to 50 per cent of the data (interquartile region). Above and below the boxes there are two vertical lines, called the whiskers. The line above the box ends at a value which is 1.5 times the interquartile range (i.e. the vertical height of the box) from the upper edge of the box. Similarly, the line below the box ends at a value which is 1.5 times the interquartile range, from the lower edge. Each whisker is truncated at a WD mass value belonging to a particular data point. Since there may be no data point whose value is exactly 1.5 times the interquartile distance, the whisker may be shorter than its nominal range. This is the case for the line below the boxes. For some boxes, the lower edge is very close to the end of the vertical line below the box. This is because there are no data points below this. Any points that lie outside the range of the whiskers are considered outliers. Each cloud of points to the left of each box represents the distribution of WD masses, and above each box the number of CVs in the models at a particular time is given. Notice that model S2 contains more high-mass WDs than model S-BSE due to dynamical exchanges that put massive WDs into the CV population. Regardless, both models are dominated by low-mass WDs after $\sim 4$ Gyr. Model K2 is dominated by massive WDs throughout the cluster evolution, although a non-conspicuous peak is observed for low-mass WDs. For more details, see Section 3.7.1.

models K2 and S-BSE). Furthermore, model S2 (a dynamical environment) has slightly more high-mass donors than model S-BSE (a field-like environment). This is because the greater is the WD mass, the greater can the donor mass be in order for the stability limit to remain respected. Since dynamically formed CVs tend to have greater WD masses, we expect greater donor masses as well. In the beginning, the boxes in Fig. 7 are very narrow and have mainly high-mass donors, for all models. After $\sim 3$ Gyr of evolution, the median drops below $0.1 \mathrm{M}_{\odot}$ in models S2 and S-BSE. In model $\mathrm{K} 2$, however, the median remains above this value.

At $\sim 4$ Gyr, the median mass for all models is below $0.1 \mathrm{M}_{\odot}$. After that, the distributions for models S2 and S-BSE become completely dominated by extremely-low-mass donors, with a huge peak around the first quartile and a long tail toward higher masses. This feature remains up to a cluster age of $12 \mathrm{Gyr}$. The long tail is caused by the constant addition of CVs to the population, and the huge peak by $\mathrm{CV}$ evolution. 
Belloni et al.
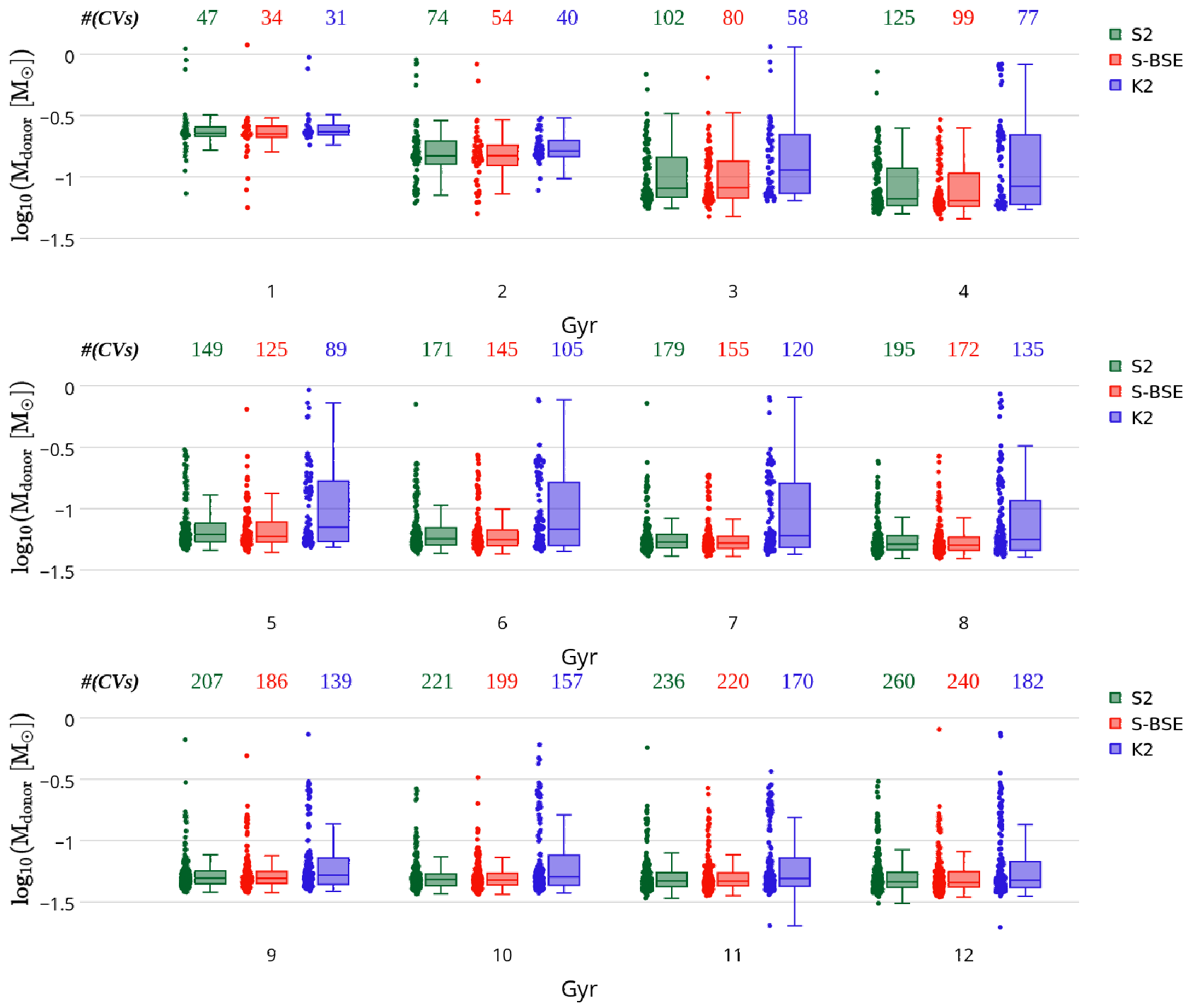

Figure 7. Evolution of the donor mass distribution in time for all CVs in 2 out of the 6 models. The notations are the same as in Fig. 6. Notice that the donor masses decrease with time not only via the addition of new systems, but also due to CV evolution (mass transfer). Model K2 has slightly more massive donors than models S2 and S-BSE. For more details, see Section 3.7.2.

On the other hand, model K2 maintains a bimodal distribution from $\sim 4$ Gyr up to $\sim 7$ Gyr. At this point $(\sim 7 \mathrm{Gyr})$, the $\mathrm{CV}$ population in this model has more than 50 per cent of the entire CV present-day population (at $12 \mathrm{Gyr}$ ). After $7 \mathrm{Gyr}$, the CV population has a similar feature to the other models: a huge peak around the first quartile, and a long tail towards higher masses. Notice that, at $\sim 8 \mathrm{Gyr}$, the $\mathrm{CV}$ population in model $\mathrm{K} 2$ is dominated by extremely-low-mass donors, and the addition of more massive donors is not able to further maintain the bimodal shape of the distribution.

Interestingly, we can clearly see in the boxes (at 11 and 12 Gyr) for model K2 the CV mentioned in Section 3.5.2, which had its evolution accelerated by a strong dynamical interaction. Note that, in the figure, such a CV donor appears as an isolated point below $\log _{10}\left(M_{\text {donor }}\right)=-1.5$, which is a visualization of the effects of strong dynamical interactions that accelerate $\mathrm{CV}$ evolution. Although such objects are quite interesting from a dynamical perspective, they are rare as already pointed out and probably part of the faintest population of CVs in GCs, which makes them unlikely to be observed.

\subsubsection{Discussion on the age-dependence of CV properties}

Importantly, it is not just CV evolution that causes the decrease in the median donor mass in the overall population in models S2 and S-BSE. Additionally, more low-mass CVs are added to the population as time goes on. Again, this is associated with the turn-off mass evolution, which makes low-mass WDs appear more frequently at late times. We showed in Section 3.3.4 that the CV formation rate is driven by the WD formation rate in the cluster, and the time delay needed for WD-MS binaries to become CVs. We further showed that the time delay is small in comparison with the cluster lifetime. This implies that we should, indeed, expect the addition of more low-mass $\mathrm{CV}$ s to the entire $\mathrm{CV}$ population, relative to higher mass CVs.

On the other hand, in model K2 (i.e. for dynamically formed $\mathrm{CVs}$ ), we find that the WD mass distribution is dominated by high- 
mass WDs. Additionally, even though the newly added CV donors in model $\mathrm{K} 2$ are more massive than the newly added $\mathrm{CV}$ donors in models S2 and S-BSE, we still find that after $\sim 8 \mathrm{Gyr}$, the CV population is predominantly composed of extremely-low-mass donor, which is caused mainly by mass loss due to CV evolution.

Having described the overall picture for the age-dependence of $\mathrm{CV}$ properties, we now turn to a discussion of the implications of these features for $\mathrm{CV}$ populations.

The donor mass evolution is very important for determining the CV status at any given time. In the case of cluster CVs, we clearly see from Fig 7 that the majority of CVs have extremelylow-mass donors after $12 \mathrm{Gyr}$ of cluster evolution. This means that they are much fainter than the Galactic field $\mathrm{CV}$ s as a whole, if one assumes that the star formation rate in the Galaxy is roughly constant and bears in mind that star formation in GCs took place only when the clusters formed. Thus, we expect that cluster CVs should have donors with lower masses than the observed Galactic field CVs.

This brings serious restrictions to any comparison between predicted cluster CVs and observed field CVs. The old field CVs are still missing, although a few of them have now been detected (e.g. Littlefair et al. 2006; Hernández Santisteban et al. 2016). Regardless, the majority of the observed CVs in the field seem to be much younger than GC CVs.

Ak et al. (2015) inferred kinematic ages in a sample of field CVs and concluded that 94 per cent of CVs in the solar neighbourhood belong to the thin-disc component of the Galaxy. Mean kinematical ages of $3.40 \pm 1.03$ and $3.90 \pm 1.28 \mathrm{Gyr}$ were found for the non-magnetic thin-disc CVs below and above the period gap, respectively. Some GC CVs can be up to 4 times older than the observed field CVs. This means that comparing GC CVs and field $\mathrm{CVs}$ should be done carefully, especially if one considers predicted GC CVs and observed field CVs.

\subsection{Destroyed CVs}

Thus far, we have described CVs formed in clusters that survive until the present-day. In this section we deal instead with CVs that do not survive, and are not found in the present-day clusters. These are called 'destroyed' $\mathrm{CVs}^{2}$, in the sense that they are formed but do not survive until a cluster age of $12 \mathrm{Gyr}$.

Belloni et al. (2016, see section 3.5.1) describe the three main destruction channels associated with cluster CVs. These are: (i) destruction due to binary stellar evolution (the $\mathrm{CV}$ stops being a $\mathrm{CV}$ due some evolutionary process and without any influence from dynamical interactions); (ii) destruction due to escape (the CV life in the cluster is interrupted because the $\mathrm{CV}$ escaped the cluster, independent of whether or not the $\mathrm{CV}$ remained a $\mathrm{CV}$ post-escape); and (iii) destruction due to dynamical interactions (the CV death was caused by a dynamical interaction, but the outcome of this interaction remains in the cluster).

In what follows, we describe the main features associated with the above destruction channels. The number of CVs that do not survive until $12 \mathrm{Gyr}$, separated according to their main formation and destruction channels, are given in Table 2.

2 The reader should keep in mind that the term 'destroyed' used here is not necessarily associated with a destructive process stricto sensu. The term adopted here has a more general sense: $\mathrm{CVs}$ that are created during the cluster evolution but do not survive in the cluster up to the present-day, which can occur for many reasons.
Table 2. Number of CVs that are formed during the cluster evolution, but are not present in the PDP, separated according to their main formation and destruction channels. We also indicate the number of CVs for which exchanges are the main dynamical process involved in their formation.

\begin{tabular}{lcccccccc}
\hline Model & \multicolumn{3}{c}{ Formation Channels } & \multicolumn{3}{c}{ Destruction Channels } & Total \\
\hline & BSE $^{\mathrm{a}}$ & WDI $^{\mathrm{b}}$ & SDI $^{\mathrm{c}}$ & Exchange & DBSE $^{\mathrm{d}}$ & DESC $^{\mathrm{e}}$ & DDI $^{\mathrm{f}}$ & \\
\hline \hline $\mathrm{K} 1$ & 45 & 43 & 4 & 1 & 89 & 3 & 0 & 92 \\
\hline $\mathrm{K} 2$ & 29 & 111 & 272 & 144 & 398 & 3 & 11 & 412 \\
\hline $\mathrm{K} 3$ & 18 & 69 & 211 & 102 & 277 & 10 & 11 & 298 \\
\hline $\mathrm{S} 1$ & 114 & 2 & 0 & 0 & 97 & 19 & 0 & 116 \\
\hline $\mathrm{S} 2$ & 169 & 131 & 24 & 11 & 324 & 0 & 0 & 324 \\
\hline S3 & 7 & 23 & 18 & 14 & 33 & 14 & 1 & 48 \\
\hline \hline
\end{tabular}

a Binary Stellar Evolution

b Weak Dynamical Interaction

c Strong Dynamical Interaction

d Destruction due to Binary Stellar Evolution

${ }^{\text {e }}$ Destruction due to Escape

${ }^{\mathrm{f}}$ Destruction due to Dynamical Interaction

\subsubsection{Unstable CVs}

From Table 2, we see that the most prominent destruction channel is unstable mass transfer that leads the $\mathrm{CV}$ to merge, which is in good agreement with Ivanova et al. (2006) and Shara \& Hurley (2006). This happens because the WD cannot stably burn the accreted material (Nomoto et al. 2007), which causes even higher mass transfer rates, and, subsequently, a CEP followed by a merger.

Considering all six models, more than 95 per cent of the destroyed CVs are destroyed because of unstable mass transfer. In the Kroupa models, they are mainly formed due to strong dynamical interactions. In the Standard models, they are mainly formed due purely to a CEP.

The reason for the unstable mass transfer is the high mass of the donor, which is much greater than the WD mass. This implies that the $\mathrm{CV}$ mass ratio is much larger than unity. The critical mass ratio that separates stable and unstable mass transfer can be obtained by equating the adiabatic mass-radius exponent and the mass-radius exponent of the donor Roche-lobe. Such a critical mass ratio is of order unity for almost all donors, if one assumes the classical version of the angular momentum loss prescription (Schreiber et al. 2016, for more details). Thus, CVs with very massive donors $\left(M_{\mathrm{donor}} \gtrsim M_{\mathrm{WD}}\right)$ will probably undergo another CEP, and merge.

It is important to note that few $\mathrm{CV}$ s with $M_{\text {donor }} \sim M_{W D}$ will evolve towards stable mass transfer. In all six models, we detected only one such case. CVs undergoing unstable mass transfer live only for a few Myr.

\subsubsection{Escaping CVs}

Anther possible mechanism for destroying $\mathrm{CV}$ s is their escape from the cluster, due either to relaxation or direct (strong) dynamical interactions. Escape due to relaxation takes place gradually, and the $\mathrm{CV}$ probably remains as a $\mathrm{CV}$ after the escape. On the other hand, escape due to dynamical interactions is a more violent process; the binary typically has its orbital parameters changed considerably, and the $\mathrm{CV}$ is probably no longer a $\mathrm{CV}$ after the escape.

From Table 2, escapers correspond to roughly 3.3 per cent of all destroyed CVs. Most of the escapers escape due to relaxation (94 per cent). They are mostly stable CVs, in general, before they 
escape. This means that, had they not escaped, they would remain as CVs in the cluster.

Unstable CVs, with high-mass donors, tend to have longer periods than stable CVs. Consequently, they are more likely to suffer a strong dynamical interaction. On the other hand, they have short lives, which means that they do not have much time to interact.

\subsubsection{CVs destroyed by Dynamical Interactions}

Finally, some CVs can literally be destroyed by dynamical interactions. Since the CVs tend to be very hard binaries, the probability of such destructive interactions is small (Leigh et al. 2016). Indeed, in all six models, only 1.7 per cent of the destroyed CVs were destroyed by strong dynamical interactions. As with the escapers, these were stable $\mathrm{CV}$ s before the interaction occurred.

Above all, only 5 per cent of all destroyed CVs are stable. This means that once a stable CV is formed, it tends to live for a long time, unless it escapes from the cluster or has its orbital parameters changed by a dynamical interaction.

\subsection{CVs formed from escaping binaries}

In this last section, we discuss CVs that form from escaping binaries, ejected sometime within the $12 \mathrm{Gyr}$ of cluster evolution. We stress that the escaping binaries escape at any time and all CVs were evolved from the time of escape up to 12 Gyr using the BSE code.

As expected, no CVs escaped from model $\mathrm{K} 1$. This is easy to understand by considering the strength of dynamical interactions characteristic of this model. The cluster is very sparse and the probability of interaction (leading to a change in the binary parameters) is small. Consequently, the probability of significant changes occurring in the escaping binaries (before the escape) is small. Since not a single $\mathrm{CV}$ is formed through pure binary stellar evolution in the Kroupa models, we see that model K1 should produce only a few (if any) CVs from the escaping binaries, since the influence of dynamics in this model is weak.

Models K2 and K3 have 12 and $23 \mathrm{CV}$ s formed from escaping binaries, respectively. In these models, future CVs were created prior to escape and, in a field-like environment, the $\mathrm{CV}$ s were able to form. Analogously to what happens for the GC CVs in models $\mathrm{K} 2$ and $\mathrm{K} 3$, CVs formed from escaping binaries tend to have high-mass WDs. This is the result of strong dynamical interactions, specifically exchanges.

Models S1 and S3 have, respectively, 34 and $19 \mathrm{CVs}$ formed from escapers. Note that this number of CVs is comparable to the number of CVs present in the cluster at the present-day (41 in S1, and 27 in S3). This is easy to understand by considering the evolution of these clusters (Section 3.3.1). Both models are post-corecollapse and are expanding due to the energy source in their cores (S1 due to a subsystem of black holes and $\mathrm{S} 3$ due to binaries). This makes the escape rate high, which in turn reflects in the number of $\mathrm{CV}$ s formed from escaping binaries.

Interestingly, model S2 produces only $4 \mathrm{CVs}$ out of all the escaping binaries. This is again connected to the cluster evolution. As already pointed out, this cluster is approaching core-collapse (which will take place at $\sim 13 \mathrm{Gyr}$ ). Consequently, the cluster is not expanding, as in S1 and S3. As such, only a few future CVs escape from the cluster.

In models $\mathrm{K} 2$ and $\mathrm{K} 3$, most of the escaping binaries that later become $\mathrm{CVs}$ escape from the cluster between $\sim 6$ and $\sim 8 \mathrm{Gyr}$.
In models $\mathrm{S} 1$ and $\mathrm{S} 3$, however, the escape time has a roughly flat distribution, with a peak at $\sim 10 \mathrm{Gyr}$.

In all 6 models, $92 \mathrm{CVs}$ formed from escaping binaries. Of those, $\sim 62$ per cent of CVs have WDs with masses greater than 0.5 $\mathrm{M}_{\odot}$. Additionally, $\sim 61$ per cent of these $\mathrm{CVs}$ are period bouncers, which is much less than the fraction of period bouncers found in the GC CVs ( $\sim 87$ per cent $)$.

In general, the fraction of escaping binaries that become CVs is $\sim 10^{-4}$. Unfortunately, due to the small total number of CVs formed from escaping binaries (and the relatively small number of simulations), we are unable to say more about them. It seems that a very small fraction of the Galactic field CVs in the halo might have their origins in GCs.

When more models of the MOCCA-SURVEY are analyzed, we will better constrain the properties of CVs formed from escaping binaries, and their relation to the Galactic field CVs.

\section{CONCLUSIONS}

In the first paper of this series (Belloni et al. 2016), we discussed six specific MOCCA models with a focus on the properties of their present-day CV populations. In this paper, we concentrate instead on a discussion of the properties of the progenitor and formation-age populations.

Our results show good overall agreement with previous investigations, both with respect to the most common CV formation channels (Ivanova et al. 2006) and the acceleration/retardation of $\mathrm{CV}$ evolution prior to $\mathrm{CV}$ formation, by (indirectly) affecting the CV progenitor binary (Shara \& Hurley 2006) .

The main results of this paper can be summarized as follows:

(i) Dynamics can extend the parameter space applicable to $\mathrm{CV}$ progenitors (with respect to CVs formed without influence of dynamics), and allow binaries that would not become CVs to evolve into CVs.

(ii) Sparse clusters have more CVs formed through a CEP, relative to denser clusters with more dynamically formed CVs. The number of dynamically formed CVs decreases with decreasing cluster density, as expected.

(iii) The WD-MS binary formation rate is characterized by an initial burst, followed by a smoothly decreasing rate. The CV formation rate shows the same initial burst, although it is followed by a nearly constant formation rate. This is caused by the timedelay between the time of WD-MS binary formation and CV formation. This is, in general, in good agreement with the findings of Ivanova et al. (2006), who claimed that the relative number of CVs that appear is roughly constant throughout the cluster evolution.

(iv) The CV formation rate can either be accelerated or retarded due to dynamical interactions, which is in good agreement with the results of Shara \& Hurley (2006). Additionally, the CV is unlikely to change after $\mathrm{CV}$ formation, i.e. after $\mathrm{CV}$ formation, it is improbable that the $\mathrm{CV}$ will be affected by dynamical interactions, because the CVs are dynamically hard binaries with small interaction crosssections.

(v) The CVs are mainly formed as short-period systems, which indicates that they will be very faint objects by the present-day.

(vi) Dynamically formed CVs tend to have massive WDs, in general, due to exchanges. This was pointed out by Ivanova et al. (2006).

(vii) The properties of CV populations change with time, which can lead to confusion upon comparing predicted cluster CVs (older) to observed Galactic field CVs (younger). 
(viii) Before the present-day, CVs can be 'destroyed' either via unstable mass transfer (most of them), dynamical interactions, or cluster escape, in good agreement with Shara \& Hurley (2006) and Ivanova et al. (2006).

(ix) Very few field CVs could have their origins in GCs.

This concludes the first part of our investigation into CV formation in GCs using the MOCCA, split between two first papers of which this is the second. Thus far, our results have shown good overall agreement with previous observational and theoretical works.

Future investigations will concentrate on the effects of the empirical consequential angular momentum loss prescription (Schreiber et al. 2016) (which is associated with the mass loss from the system), and on the CEP parameters, in deciding the predicted $\mathrm{CV}$ properties. Special attention will be given to the absence of field-like CVs in the Kroupa models. Equally interesting are the $\mathrm{CV}$ siblings (AM CVn and symbiotic stars), especially upon considering that the first AM CVn in a GC might have just been discovered in NGC 1851 (Zurek et al. 2016). We plan to extend the CATUABA code in order to include these interesting systems in order to extend our analysis to include the entire population of accreting white dwarf binary systems in GCs. After CATUABA is complete and automated, we will begin our analysis of the models of the MOCCA-SURVEY (Askar et al. 2016).

\section{ACKNOWLEDGEMENTS}

DB would like to kindly thank Christian Knigge, Mónica Zorotovic, and Matthias Schreiber for useful discussions and suggestions, which made the quality of the paper increase substantially. We would also like to thank the referee Matthew Benacquista for his comments that improved this work. DB was supported by the CAPES foundation, Brazilian Ministry of Education through the grant BEX 13514/13-0. MG and AA were supported by the National Science Centre through the grant DEC2012/07/B/ST9/04412. AA would also like to acknowledge support from the National Science Centre through the grant UMO2015/17/N/ST9/02573 and partial support from Nicolaus Copernicus Astronomical Centre's grant for young researchers.

\section{REFERENCES}

Ak T., Bilir S., Özdönmez A., Soydugan F., Soydugan E., Püsküllü Ç., Ak S., Eker Z., 2015, Ap\&SS, 357, 72

Askar A., Szkudlarek M., Gondek-Rosińska D., Giersz M., Bulik T., 2016, preprint, (arXiv:1608.02520)

Belloni D., Giersz M., Askar A., Leigh N., Hypki A., 2016, MNRAS, 462, 2950

Breen P. G., Heggie D. C., 2013, MNRAS, 436, 584

Camacho J., Torres S., García-Berro E., Zorotovic M., Schreiber M. R., Rebassa-Mansergas A., Nebot Gómez-Morán A., Gänsicke B. T., 2014, A\&A, 566, A86

Casertano S., Hut P., 1985, ApJ, 298, 80

Fregeau J. M., Cheung P., Portegies Zwart S. F., Rasio F. A., 2004, MNRAS, 352,1

Fukushige T., Heggie D. C., 2000, MNRAS, 318, 753

Giersz M., Heggie D. C., Hurley J. R., Hypki A., 2013, MNRAS, 431, 2184

Giersz M., Leigh N., Hypki A., Lützgendorf N., Askar A., 2015, MNRAS, 454,3150

Hénon M. H., 1971, Astrophysics and Space Science, 14, 151

Hernández Santisteban J. V., et al., 2016, Nature, 533, 366

Hurley J. R., Pols O. R., Tout C. A., 2000, MNRAS, 315, 543
Hurley J. R., Tout C. A., Pols O. R., 2002, MNRAS, 329, 897

Hypki A., Giersz M., 2013, MNRAS, 429, 1221

Hypki A., Giersz M., 2016a, preprint, (arXiv:1604.07033)

Hypki A., Giersz M., 2016b, preprint, (arXiv:1604.07054)

Iben Jr. I., Livio M., 1993, PASP, 105, 1373

Ivanova N., Heinke C. O., Rasio F. A., Taam R. E., Belczynski K., Fregeau J., 2006, MNRAS, 372, 1043

Knigge C., 2011, in Schmidtobreick L., Schreiber M. R., Tappert C., eds, Astronomical Society of the Pacific Conference Series Vol. 447, Evolution of Compact Binaries. p. 3 (arXiv: 1108.4716)

Knigge C., 2012, Mem. Soc. Astron. Italiana, 83, 549

Knigge C., Baraffe I., Patterson J., 2011, ApJS, 194, 28

Kroupa P., 2008, in Aarseth S. J., Tout C. A., Mardling R. A., eds, Lecture Notes in Physics, Berlin Springer Verlag Vol. 760, The Cambridge N-Body Lectures. p. 181 (arXiv:0803.1833), doi:10.1007/978-1-4020-8431-7_8

Leigh N., Giersz M., Webb J. J., Hypki A., De Marchi G., Kroupa P., Sills A., 2013, MNRAS, 436, 3399

Leigh N. W. C., Lützgendorf N., Geller A. M., Maccarone T. J., Heinke C., Sesana A., 2014, MNRAS, 444, 29

Leigh N. W. C., Giersz M., Marks M., Webb J. J., Hypki A., Heinke C. O., Kroupa P., Sills A., 2015, MNRAS, 446, 226

Leigh N. W. C., Geller A. M., Toonen S., 2016, ApJ, 818, 21

Littlefair S. P., Dhillon V. S., Marsh T. R., Gänsicke B. T., Southworth J., Watson C. A., 2006, Science, 314, 1578

Nelemans G., Siess L., Repetto S., Toonen S., Phinney E. S., 2016, ApJ, 817,69

Nomoto K., Saio H., Kato M., Hachisu I., 2007, ApJ, 663, 1269

Schenker K., Kolb U., Ritter H., 1998, MNRAS, 297, 633

Schreiber M. R., Zorotovic M., Wijnen T. P. G., 2016, MNRAS, 455, L16

Shara M. M., Hurley J. R., 2006, ApJ, 646, 464

Spitzer L., 1987, Dynamical evolution of globular clusters

Stodółkiewicz J. S., 1986, Acta Astron., 36, 19

Wang L., et al., 2016, MNRAS, 458, 1450

Warner B., 1995, Cambridge Astrophysics Series, 28

Yaron O., Prialnik D., Shara M. M., Kovetz A., 2005, ApJ, 623, 398

Zorotovic M., Schreiber M. R., Gänsicke B. T., Nebot Gómez-Morán A., 2010, A\&A, 520, A86

Zorotovic M., Schreiber M. R., Gänsicke B. T., 2011, A\&A, 536, A42

Zurek D. R., Knigge C., Maccarone T. J., Pooley D., Dieball A., Long K. S., Shara M., Sarajedini A., 2016, MNRAS, 460, 3660

This paper has been typeset from a $\mathrm{T}_{\mathrm{E}} \mathrm{X} / \mathrm{L} \mathrm{T} \mathrm{E} \mathrm{X}$ file prepared by the author. 